$\mathbb{T}$ Periodica Polytechnica Electrical Engineering and Computer Science

61(2), pp. 83-115, 2017

https://doi.org/10.3311/PPee.11067

Creative Commons Attribution (i)

RESEARCH ARTICLE

\section{BME VIK Annual Research Report on Electrical Engineering and Computer Science 2016}

\author{
Hassan Charaf, Gábor Harsányi, András Poppe, \\ Sándor Imre, Bálint Kiss, Tamás Dabóczi, \\ Gyula Katona, Lajos Nagy, Gábor Magyar, István Kiss
}

\section{Preface}

Since being established in 1949, the Faculty of Electrical Engineering and Informatics (VIK) BME has played a flagship role in the development of electronics, IT and computer science in Hungary.

We are proud of combining engineering applications with sound scientific results, which is the assurance of high-level industrial collaboration leading to novel results and innovation.

The various collaborations with our industrial partners has made it clear that the industry expects methods and results which make their industrial processes more effective and increase productivity and quality. Thus, participation in these collaborations give a competitive edge and ensure the continuous development of VIK. These factors have positioned our Faculty as a significant source of knowledge transfer and a treasured partner in various cooperation activities.

The current paper gives a brief account of the results achieved at the Faculty of Electrical Engineering and Informatics in the year 2016 and, at the same time, tries to encompass the research activities conducted at different departments of the Faculty.

We believe that this survey proves to be an informative summary about our scientific and technological contributions made in the year 2016.

László Jakab (dean, BME VIK) János Levendovszky (vice-dean in charge of scientific affairs, BME VIK)

\section{Department of Automation and Applied Informatics}

Department of Automation and Applied Informatics (AUT) is one of the largest and dynamically developing departments at BME with diverse scope competences such as control theory, embedded systems, software modelling, applied software development, Internet of Things, power electronics, mechatronics, and many others. The department's main activities are education, research and development. Training versatile electrical and software engineers with solid practical knowledge is our top priority. Our profile also includes developing high quality software and hardware solutions for industry partners. All of our activities are backed by strong research background.

In 2016, the department organized and hosted a number of domestic and international events. In cooperation with the industry, AUT has organized the IoT4U conference. It was dedicated to Internet of Things, cloud and data management as well as the integration of IoT into various formats. RobonAUT, the yearly domestic amateur robotics contest was organized by the department in February 2016. The yearly contest is open to teams of students, organized either in student projects within the department or independent research teams.

The next sections summarize the key research results of the department in year 2016 .

\subsection{Software Development Methods and Mobile Platforms}

The diversity of mobile platforms necessitates the development of the same mobile application for all major mobile platforms, what requires considerable development effort. Mobile application developers are multiplatform developers, but they still prioritize the platforms, therefore, not all platforms are equally important for them. Appropriate methods, processes and tools are required to support the development in order to achieve better productivity.

The research on educational mobile applications that adapt to the mental state of the user required a method for estimating the difficulty of tasks found in the serious games. There are generated or random assignments where the effort required from the user is not known or cannot be estimated in advance. 
A good example for such a quiz is adaptive figural abstraction tests like Raven's progressive matrices with generated exercises [1]. We propose a solution to this problem by presenting a model of an algorithm for game task difficulty estimation based on Bayesian probability theory and existing research on human intelligence. We have also introduced a simulator which is used to analyze the validity and efficiency of the estimation algorithm [2].

Regarding software development, we worked out a method of platform-independent intermediate-level obfuscation that can be used in various fields of information security. The offered methods and approaches have been demonstrated in IL Obfuscator tool [3].

\subsection{IoT and Smart City}

The Internet of Things (IoT) is transforming the surrounding physical objects into an ecosystem of information that enriches our everyday life. Novel application and service development methods and frameworks are required to support the realization of solutions covering data collection, transmission, and data processing, analysis, reporting, and advanced querying. Our SensorHUB framework utilizes the state-of-the-art open source technologies and provides a unified tool chain for IoT related application and service development. SensorHUB is both a method and a research accelerator environment to support IoT related application and service development $[4,5]$. The framework is worked out in the context of EIT (European Institute of Innovation \& Technology) Climate-KIC [6] SOLSUN project.

The SOLSUN (Sustainable Outdoor Lighting \& Sensory Urban Networks) project is about to demonstrate how intelligent city infrastructure can be created in a cost-effective and sustainable way by reusing existing street lighting as the communications backbone [7]. We apply different technologies and methods to reduce energy consumption at the same time as turning streetlights into nodes on a scalable network that is also expandable for other applications. Sensors capture data on air pollution, noise pollution, and traffic density [8]; gathered information is used to address traffic congestion, a key contributor of GHG emissions in cities.

SOLSUN project develops an integrated technology platform where several components of the SensorHUB framework and the knowledge of the SensorHUB team is utilized. The project brings together a strong core of public, private, and academic partners with the combined expertise to develop outcomes that can be exploited on a global scale. The project is carried out between 2015 and 2017 by the following partners: Select Innovations Limited (UK), British Telecommunications Plc. (UK), Municipality of the City of Budapest (Hungary), PANNON Pro Innovation Services Ltd. (Hungary), and Budapest University of Technology and Economics (Hungary).

Collected data is considered one of the key parameters controlling smart systems around the city. Furthermore, data is important for the study of the urban environment of the city. For example, burn hours and energy consumption can be adjusted to meet the sufficient needs of warmer areas over colder ones or over the same areas frequently due to land use and land cover changes; further, there obviously exist facts of reduced energy and more rapid runoff of $\mathrm{GHG}$ emissions.

The challenges for this research is to choose the appropriate statistical tool for urban data. Thus, we need to do an exploratory analysis to determine factors such as means, maxima, and quantiles. Our results showed 32 associations between containers and measured values. The container, located in "Beccles Road", measures the highest temperature and consumes the highest lamp current. Regardless of which location, the analysis showed that there is a strong correlation between temperature and lamp current. In other words, the higher temperature scores, the much lamp current consumed. The "Beccles Road" location also scores high reading in PSU voltage and energy in another container.

The cv4sensorhub project aims at the extension of SensorHUB with a semi-automatic image-processing framework. On one hand, it provides data model and operations for several image-processing techniques, and on the other hand, it includes customizable user interface tools. The user interface is meant for applications where the user needs to supervise the image processing either to check the automatic results, or to manually guide the smart tools in cases, which are too complex for fully automatic approaches. The long-term goal of the project is to support the development of interdisciplinary applications related to image processing.

Currently, the framework has two applications built over it [9]. The GrainAutLine [10] system helps experts to analyze marble thin section images for marble provenancing. The ChemoTracker application automates white blood cell migration tracking in microscopy images to support immunology research.

The research project titled Joint Design of Communication and Control for Connected Cars in 5G Communication Systems was conducted in cooperation with the 5GLab at TU Dresden and Nokia Bell Labs. The goal was to investigate the capabilities of autonomous and connected cars and study the influence of various parameters on the traffic efficiency of communicating cars. We developed a physical testbed, which was presented at multiple exhibitions, including the Mobile World Congress (MWC) 2016 in Barcelona and Shanghai, and the $5 \mathrm{G}$ Connect Congress in Munich to highlight the importance of connected cars over autonomous cars and their role in traffic efficiency [11].

The testbed comprises a complete architecture of a connected car scenario, including communication-capable miniature cars driving on a street with intersections. The testbed also includes a traffic coordination software running as a cloud service, ensuring that there are no crashes in the streets, especially in the intersection even in the presence of human interaction. For 
instance, if one of the cars is manually stopped on the street the other cars react to that "in time" preventing any accidents.

\subsection{A Hypothesis Model for Vehicle Tracking}

Counting traffic more and more efficiently is a major problem of the field of urban design. The more precise knowledge, and expected amount of traffic can be used to design more attractive, functional, and sustainable junctions, thus leading to an environment-friendly and closer-to-optimal urban design. Since the whole process of tracking can be separated into two mayor steps, it is better to investigate them independently. These two steps are detection and tracking. In detection, an object is found and descriptors are extracted, while in tracking these detections are linked based on physical parameters and descriptors.

Compared to general tracking methods, our architecture has a few significant differences. The main concept is that the final output of the tracking is a graph, where each node is generated by the tracker, and has a probability value assigned to it.

The built graph is called hypothesis graph. The resulting hypothesis graph is a tree, having more and more branches and leaves with time, but no merge. Since parent chain is always trivial, a final probability value of a hypothesis can be calculated as accumulated certainty of its nodes.

After building the whole hypothesis trees, all the obtainable information is created to make the final decision whether it gets in the set of counted vehicles or should be dropped. We get rid of inviable hypotheses, which have improbable attributes, e.g., too high velocity, impossible route, etc. However, all the remaining hypotheses are appropriate and many of them are related to the same vehicle. The idea of duplicate filtering, which is based on the fact that there cannot be multiple vehicles at the same place simultaneously, could be more accurate, if not only the rate of the covered frames were taken into consideration, but also their weight based on the reliability of their situations. A vehicle with greater angle to the target is covered more often, while a vehicle viewed from top view is never covered, and reveals duplicates instantly.

Our measurements and results were impressive compared to regular tracking methods, especially in cases, when vehicles became occluded or have been stopped for a long time. The results are now being in the process of publication, and will be available soon in conference proceedings and journals.

\subsection{Network Coding Enhanced Data Distribution}

In our work, we are investigating the possibilities to create peer-to-peer (P2P) assisted video on demand (VoD) streaming with network coding [12]. We propose a BitTorrent-like protocol for data dissemination. We are using only JavaScript to create an application that can run in any browser without any third-party plugin. The network contains a content server that is always available like in case of any streaming services. The clients are capable of creating browser-to-browser connections with WebRTC, as well as caching and exchanging network coded data. Through measurements, we show that employing our protocols for data-streaming, average data download speed can be significantly increased and server load can be decreased up to $80 \%$. To validate the scalability of our solution, we tested our protocols in a network with more than 100 tablets to deliver smooth video playback [13].

We also investigate the possibilities of creating a Mobile Edge Cloud (MEC) [13-15]. To achieve sub millisecond latency, bringing the services closer to the clients is inevitable. The aim of MEC is to move the clouds to the edge of the network, close to the clients or even move the clouds with clients. This technology is crucial in case of connected cars, where the cars are connected to a cloud-based service. Keeping the service in a centralized place increases the latency that can lead to accidents, so the services have to travel with cars. In our work, we propose a protocol and architecture, called Agile Cloud Migration (ACM). We also implemented a working version of ACM in a small tron-like 2D game. The players are able to control their in-game avatars, while the game server can be migrated live from part of earth to another, or to the edge, close to the players, reducing the latency to nearly zero.

A crucial problem limiting the widespread use of erasure coding in distributed storage lies in the high network cost of rebuilding lost data. A theoretical model was created and lower bounds on this cost was introduced in [16]. This suggests that network coding is ideal for highly dynamic, decentralized storage.

Previous research $[17,18]$ have shown that using online network coding with recoding capabilities, the latency is significantly decreased. Our current aims lie in minimizing the damage recoding does to online encoding while retaining the gains it provides [19].

We also employ machine-learning approaches for the prediction of header compression utilities [20], which will enable the compression to dynamically adapt to varying channel conditions. This will ensure that the compression always performs at best efficiency.

By assuring the optimal utilization of the proposed solutions, we enable future wireless mesh networks for the adoption of low latency applications, such as automated vehicle steering, surveillance, live multimedia transmission, etc. [21-24]

\subsection{Mechatronics}

With regard to mechatronics area, we can report both practical and theoretical results.

Within the framework of a cooperation between AUT and AUDI Hungária Motor Kft. started in 2011, we have developed a novel test method for fast and accurate measurement of iron losses in high-speed permanent magnet synchronous machines. In addition, a dedicated laboratory system for performing automatic measurements was designed and built. Among others, the laboratory system is capable of measuring the iron loss vs. 
frequency as well as stator and rotor temperatures. The system is capable to separate the iron loss into hysteresis and eddy current components and to correct the change in the magnetic flux density due to varying temperature of the rotor permanent magnets. The temperature of the permanent magnets embedded into steel of the rotor is measured by a novel, contactless method. The system has been jointly patented by Audi AG and AUDI Hungária Motor Kft. The description of the system and scientific results are summarized in [25].

In a theoretical work, we examined the potential realizability of photon-coupled, photo-switchable protein-based computing architectures [26]. We showed that photon coupling provides a stronger, more stable and robust integration of molecules into computing circuits than the extensively used Coulomb-coupling. The simulations suggest that circuits based on such molecules may enable the realization of nanoscale computing processors with terahertz-frequency operation.

\subsection{Summary}

BME AUT believes that innovation is the result of teamwork, when scientists and engineers work together, and creativity is the result of drawing inspiration from several sources. The real power is the harmonized team, members from various fields with different background and experience, working together on a specific problem. This motivates us to utilize the knowledge and capabilities of scientists and engineers from various fields of computer sciences (modelling, simulation, software design, development methodologies, data analyses, architecture, and others), i.e. let them work together to inspire each other and support the innovation.

\section{Department of Electron Devices}

Research and development activities at the Department of Electron Devices traditionally are connected to design of integrated circuits and novel active devices and have a special focus on thermal \& reliability issues of electronics, microelectronics, photovoltaic devices and solid-state lighting; on MEMS and smart system integration including practical realization of CPS and IoT applications. In 2016, these activities were supported by an EU FW7 project and two H2020 projects (EuroCPS, Delphi4LED - see later) and three different OTKA projects. The following subsections provide details of the most important topics and results achieved.

\subsection{Optimized RTL Design by Means of Algorithmic Formal Languages}

Nowadays the focus of digital system design seems to be shifting towards System-on-Chips equipped with run-time configurable, application-specific macrocells. Numerous tasks have to be taken over from the instruction-set microprocessors in order to cope with the ever-increasing performance requirements, while the design effort has to be kept low to handle time-to-market pressure and reduce design cost.
In the framework of this research novel solutions for digital system modelling and synthesis are being investigated. The central demand placed upon the target method is to simultaneously ensure the productivity and the possibility of detailed architectural optimizations. These somewhat conflicting requirements are well-known by the existing approaches but they are usually handled in a mutually exclusive manner; HLS (High Level Synthesis) tools are used when the development time is the primary objective and detailed hand-crafted RTL (Register-Transfer Level) modelling is applied in case of highly timing-sensitive and/or power-critical designs. To find a common ground for the contradictory needs, a novel abstraction level (Algorithmic RTL) and a formal language (Algorithmic Microarchitecture Description Language, AMDL) have been introduced [41, 49]. Using the proposed technique, the designer may describe the behaviour and define the micro-architectural details, in a unique algorithmic language environment. An AMDL-VHDL synthesis method has also been developed, which ensures the compatibility with the traditional digital system design flow. Based on the design efficiency investigations performed so far, it may be concluded that the proposed modelling means is a promising candidate for fulfilling the gap between HLS tools and hand-optimized RTL.

\subsection{Thermal-aware Design of Complex Digital ICs}

As the dissipation density reached the limits of applied air cooling solutions, thermal issues became bottlenecks in the design process of VLSI and ULSI circuits. Due to the large computational requirements, electro-thermal simulations are not applicable methods for analyzing the temperature dependent behavior of complex digital ICs. The main goal of this research is to develop new simulation methodologies to co-simulate the thermal/functional behavior of the system at different stages of the design process. Logi-thermal simulation paradigm addresses the electro-thermal simulation's issue and reduces the computational overhead by raising the abstraction level of the circuit from transistor level to gate level, RTL and above. Previous implementation of this simulation paradigm could accommodate exclusively standard cell descriptions of the circuits. Our latest results targeted the logi-thermal simulation of RTL descriptions [42]. By raising the abstraction level, and simulate only the critical path on gate level, the simulation time could be significantly reduced, while the error of the calculated temperature dependent delay was less than 3\% [42].

SoCs contain not only digital modules, but several analogue/ mixed signal and even RF circuits as well. The thermal behavior of these system can be captured by the traditional electro-thermal simulations. Our research objective is to extend the current logi-thermal simulation method with mixed-signal capabilities, thus, besides plain SystemC circuit descriptions allowing the application of SystemC-AMS. 


\subsection{Design of Cyber Physical Systems and loT Applications}

Besides development of high abstraction level modelling and synthesis methods [41] aimed at the design of processing elements to be used in CPS and IoT applications, with the support of the EuroCPS project (www.eurocps.org), in cooperation with two Hungarian SMEs we have been dealing with two projects aimed at immediate industrial applications. One targeted smart sample labelling and sample handling in biomedical testing laboratories (the SmartLAB project) while the second industrial project was aimed at the development of smart LED based street lighting luminaires with advanced built-in self-diagnostics and temperature controlled smart dimming based on an embedded multi-domain LED model (the SmartSSL project).

\subsection{The SmartLAB project}

High throughput medical diagnostics laboratories and biobanks have to face the growing amount of biomedical samples processed and stored locally. The continuous quality assurance of the even tens of thousands sample test tubes during processing, transportation and storage generates the need for comprehensive solutions. The Department of Electron Devices partnered with an innovative Hungarian SME for the SmartLAB project to develop a novel solution which uses RFID tagged test tubes and sample holders. The touchless and highly flexible technology enables the reliable and secure identification of the test tubes in the entire laboratory ecosystem, reducing the risk of sample loss or misprocessing and shortening the time of providing results for the patients. The SmartLAB system (see Fig. 1) utilizes coordinator modules in laboratory and refrigerated sample storage areas operated by Intel's embedded Edison platform.

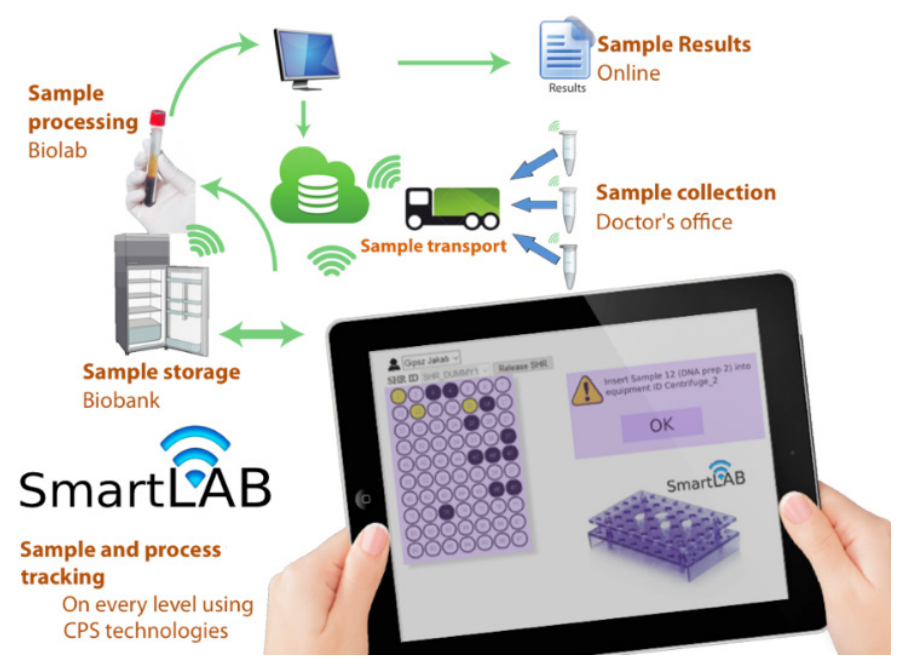

Fig. 1 Usecases of the application of the SmartLAB system

\subsubsection{The SmartSSL project}

Smart systems - according to a simplistic definition - provide new qualities and new functionalities through the integration of formerly distinct functions, components and networks. Street lighting (as other lighting applications) recently went through a drastic change offered by "LEDification". The easy electronic controllability of LEDs as light sources triggered a new change of paradigm: integration of LED based luminaires into smart systems. Smart integration necessitates among others both adaptability to the environmental conditions and intelligent remote control that require a communications systems based on a multi-layer data transfer protocol. In the SmartSLL project we aim to develop a "future proof" solution which is not restricted to lighting control but allows public lighting installations to play key roles in other smart city functions needed in outdoor public spaces such as communications with vehicles [28]. The result of the development is a new IoT device which can be installed in street lighting luminaires and allows physical layer (e.g. PLC, WiFi, ZigBee or other RF transmission) independent data transfer. The main functionality of the application layer of the protocol remains of course lighting control which is best implemented on the basis of existing standards (such as DALI), but the system allows the implementation of other communications tasks based on other application layer protocols. In this application we also used Intel's Edison platform which provides sufficient resources also to implement a multi-domain LED model which allows to exploit the temperature dependence of the efficiency/efficacy of LEDs. With such a model based control we can implement a so called iso-flux control scheme which results in constant luminous flux output of the luminaire by adjusting the LEDs' forward current to balance the changes of the ambient temperature [29,30].

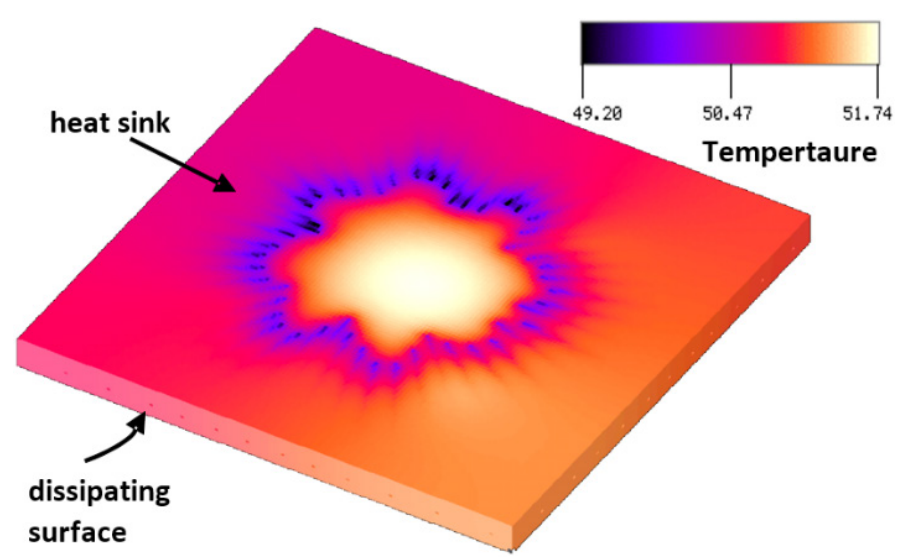

Fig. 2 Thermal simulation results of our new microchannel based cooling system simulated with the help of a recently developed compact thermal model of microchannels 


\subsection{Thermal Management and Reliability}

\subsubsection{Design and realization of integrated microchannel based cooling solutions}

In case of microscale heat sink structures which are the integral parts of modern chip or package level cooling concepts, many fabrication steps have to be fully developed before a successful chip-level cooling system is ready to be used. Recently we developed and presented a refined manufacturing technology that allows integration of microchannel based cooling solutions with IC chips, 3D stacks of chips or with solar cells [31]. Besides the new heat sink structures we also further developed the characterization method aimed for such cooling devices. The method is based on thermal transient measurement followed by structure function analysis. Our recently improved characterization method is able to account for possible non-idealities of the heat transfer processes [43]. A new method was also introduced to create a ladder-type analytical thermal model of different microscale channel based heat sink structures which allows to determine the local heat the temperature distribution along the channel depending on the channel geometries (see Fig. 2), the thermal properties of the fluid and the wall temperature(s). This model was implemented in a conventional thermal field solver to augment the capability of simulating the thermal impact of integrated heat sink. This allows the study of the operation of system-on-package (SoP) devices by electro/logi-thermal simulation [32, 42, 43].

\subsubsection{Study of thermal reliability of power semiconductor devices}

Solid-State Lighting, e-mobility and high voltage DC distribution networks impose great demand for reliable power semiconductor devices such as power diodes, power LEDs, power MOSFETs and IGBTs. As transferred energy is on a rise all application fields, every year the challenge is getting bigger for the manufacturers to increase the power rating and load current capability of their devices without sacrificing reliability. In reliability testing power or temperature cycling is used as stress method which is regularly interrupted and is followed by laboratory testing using X-ray microscopy, acoustic microscopy or device cross-sectioning. These test methods often cannot reveal the real root-causes of device aging manifesting in increased thermal resistance of a given section of the junction-to-ambient heat flow path. Degraded sections of the main heat flow path can be identified by the so-called structure function analysis of the device package in a non-destructive manner. Structure function analysis (following the thermal transient testing method) during power cycling is the basis of the in-situ method that was elaborated at the Department of Electron Devices within the NANOTHERM EU FW7 project (www.project-nanotherm.com). A versatile test environment controller hardware was designed and implemented to integrate power/temperature cycling with the non-destructive failure analysis method offered by the thermal transient measurements in an automated way [33]. This test system was also successfully used for assessing the thermal performance of newly developed Wafer Level Fan Out packaging technology [34].

\subsubsection{Thermal and multi-domain modelling of LEDs and LED applications}

In this field, we started investigating how compact thermal modelling can be applied to system level investigations of solid-state lighting solutions. As a first step, we proposed a methodology aimed at compact thermal modelling of LED luminaires. Our previous multi heat-source characterization technique was applied to LED luminaires and was combined with our prior LED multi-domain models [35, 36]. This allows luminaire level "hot lumens" calculations under arbitrary thermal conditions. The LED multi-domain measurement and modelling techniques originating from our department contributed to the successful definition and launch of a new H2020 ECSEL project titled Delphi4LED (www.delphi4LED.org) $[37,38,45]$.

\subsection{Realization and Characterization of Lab-on-a- Chip Micro-fluidic Devices}

The activity of the department in the field of biomedical devices covers a wide portfolio from model development to device design. A novel reduced order model (ROM) was constructed for the investigation of enzymatic processes encapsulated in micro droplets. This model provides two orders of magnitude decrement in the design loop time compared to time required when the conventional numerical solvers are used in the design process. BME DED and spinoff company Spinsplit LLC develop a new microchip reactor platform for fast and efficient investigation of biocatalytic, especially enzymatic processes. In a strong cooperation with the Department of Organic Chemistry and Technology of BME, the biomedically relevant PAL (EC 4.3.1.24) enzyme was successfully characterized in chip microreactor immobilized onto magnetic nanoparticles.

The so called MagneChip platform developed recently is a novel Lab-on-a-Chip (LoC) microreactor platform comprises of micro-sized magnetic reaction cells capable of anchoring biocatalyst-coated magnetic nanoparticles (MNPs) addressable and selectively. The system consists of a sensitive built-in magnetometer to quantify the entrapped particles and an in-line flow cell spectrophotometer to characterize the enzymatic process. In complex microfluidic systems including a plurality of such cells, any order of cascades of differently functionalized MNPs in serial or parallel arrangement is feasible, which allows to design highly flexible and "programmable" execution of multi-enzyme processes. This highly flexible approach was utilized in various measurements targeting otherwise challenging enzymatic characterizations. A summary of this work was published in a book chapter [48]. 


\subsection{Measurement and Modelling of Solar Cells}

As part of a long-term activity resulting in a $\mathrm{PhD}$ dissertation [51] we carried out investigation of CIGS solar cells with a vibrating capacitor Kelvin probe. The high resolution (100 um) surface potential maps were acquired under illuminated and dark conditions, thus a surface photovoltage map could also be derived. The measurement method was able to detect invisible shunts and short-circuited cells, thus giving a contactless detection possibility for such defects [46]. Results were verified by photoluminescence and dark lock in thermography measurements. The surface potential map can be also used for the detection of degradation due to ageing. Such measurements complement the techniques mentioned before in Subsection 2.4.2.

\subsection{Thermal Electric Logic Circuit (TELC) for Nanoelectronics}

We are proposing a new active thermal-electronic device family. These devices operate by means of thermal (or hot electron) coupling between adjacent domains containing heating (input) and thermally sensitive (output) elements. The theoretical background, basic equations and comparison with the conventional electron devices are the main subject of this work. According to the theoretical assumptions, the realization of the thermal-electronic device needs a very sensitive output element (temperature sensor). Among others, the metal-insulator transition (MIT) based resistor fulfils this requirement. The MIT resistor itself has thyristor-like I-V characteristics due to the high electric field and/or Joule heating and extremely strong step-like resistance drop at a given temperature. Using thermally coupled MIT and/or other resistors one can build a special device, namely phonsistor (=phonon transistor). This device consists of only bulk type intrinsic domains, containing significantly fewer regions, junctions, depleted layers, surfaces and interfaces compared to conventional electron devices. This kind of devices can probably be integrated together and with CMOS forming thermal-electric integrated circuits (TELC).

Recent results of our research work are: establishing the theoretical background of active thermal-electronic devices in general [39], realization of different thermal-electronic devices, successful development of a radio-frequency reactive cathode sputtering technology for $\mathrm{VO}_{2}$ deposition for MIT resistor [47], computer modelling of MIT effect and MIT based thermal-electronic devices [40].

\section{Department of Electronics Technology}

Electronics technology is one of the most rapidly progressing areas of engineering science. In this multidisciplinary field, new materials and technological procedures emerge year by year, immediately becoming an important and indispensable part of the common electronics assemblies. The innovations increasingly demand precise, fast and cheap production technologies. The Department of Electronics Technology (ETT) would like to give a short overview of the latest basic and applied researches in electronics applications and technologies, which point to innovations in the near future.

The researchers of BME-ETT participate in international R\&D projects sponsored by the European Union (FP7, H2020, Leonardo) and collaborate with numerous industrial partners (Bosch, Flextronics, Continental, etc.). Our short overview focuses on the following fields:

- surface characterization of material properties in the nano-level by using atomic force microscopy (AFM);

- an electrochemical method for characterising the microstructure of solder joints quantitatively;

- investigation of physical, chemical and electrochemical processes;

- investigating the structure of cracks inside solder joints by Computed Tomography (CT);

- characterizing of tin pest phenomenon by electrical resistance measurements;

- numerical and experimental study of Vapor Phase Soldering (VPS).

\subsection{Micro- and Nano-scale Material Characterization}

The main focus of the Nanotechnology Laboratory at BMEETT is the surface characterization of material properties in the nano-level with atomic force microscopy (AFM). Cavitation is a well-known phenomenon in the field of high-speed flows and it is generally considered undesired, sometimes even harmful process in hydraulic systems. However, there are several scientific and industrial applications where cavitation jets are used purposefully e.g. for jet cutting, underwater cleaning and for the improvement of fatigue strength of materials, etc. In the past years our department together with the Institute of Nuclear Techniques (BUTE) and Department of Thermohydraulics, Centre for Energy Research, Hungarian Academy of Sciences participated in a research cooperation which aimed to study the effects of cavitation erosion on various materials (copper, aluminum and stainless steel in particular).

We demonstrated that cavitation could be utilized as a tool to purposefully modify the surface characteristics of these materials (in other worlds increase their surface roughness in a controllable fashion) in the micro- and nano-levels $[52,53]$. The relation between the behavior of the cavitation erosion process and the geometrical parameters of the generator (e.g. nozzle diameter and sample standoff distance) was also studied in detail in order to maximize its efficiency $[54,55]$. In connection to the nano-level surface investigation with atomic force microscopy (AFM), another paper was published which demonstrated and discussed the possible application of a novel parameter called localization factor (introduced by A. Bonyár in 2012) for the characterization of the shape of surface structures [56].

Solder joints of an electronic product represent the mechanical, thermal and electrical interfaces between the individual 
components and the printed circuit board. Quality assessment of the solder joints are always in the centre of interest.

We have previously developed an electrochemical method, which is capable of characterising the microstructure of leadfree solder joints quantitatively (Fig. 3) [57]. By applying this technique, it was shown that the average surface between the tin-silver intermetallic compound (IMC) and the tin matrix is varying as the function of the quenching temperature of the solder samples. Huge tin-silver IMC plates were formed in solder samples when the liquid to solid transition occurred under longer time. In the case of samples that were rapidly solidified finer microstructure was formed [58]. The presence of tin-silver IMC plates is considered as a reliability concern, since the mechanical properties of such IMCs are known as very brittle.

In the past year, the microstructure of the solder joints of surface mounted resistors that were soldered in vapor phase soldering machine or infrared oven was observed. It was found that under general technological circumstances the plate-like structures may occur inside the solder bulk although the cooling rate of the applied soldering techniques are compliant with the corresponding recommendations.

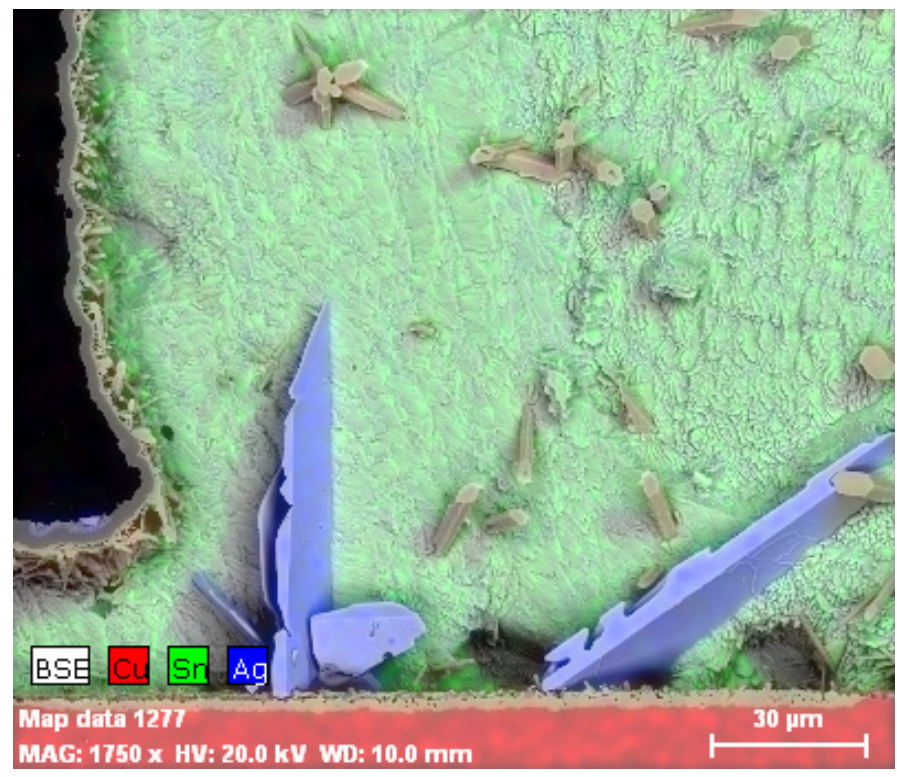

Fig. 3 Scanning electron microscope micrograph about a lead-free solder joint after the application of electrochemical etching.

The electrochemical characterization method developed at the failure analysis laboratory of the department is also suitable for detecting the presence of such platelike structures without expensive microscopy, therefore it is a promising tool for the characterization of any solder joint. The method is now being standardized in order to be applicable to every type of solder joint in different size ranges.

A method has been developed to measure the length of cracks inside solder joints, which enables the validation of Computed Tomography (CT) crack length measurements. There is a lack of information in the literature regarding the optimization of CT measurement setup; e.g., a slightly larger value of the sample rotational increment can provide acceptable resolution with much faster processing time. Thus, we developed a method and performed research about optimizing CT measurement parameters. In the experiment, cracks were formed inside $\mathrm{SAC}(\mathrm{SnAgCu})$ solder joints intentionally by aging the joints with TS test ( -40 to $+140{ }^{\circ} \mathrm{C}, 2000$ cycles) [59], and CT images were captured about them with different rotational increment $\left(1 / 4,1 / 2\right.$ and $\left.1^{\circ}\right)$ of sample projection (Fig. 4). The length of cracks was also measured with our method, which is based on capturing high-resolution radiography X-ray images about the cracks in two perpendicular projection planes [60]. The radiography results were compared to the CT measurements.

The results have proven that reducing the rotational increment increases the sharpness of the captured images and the accuracy of crack length measurements. Nevertheless, the accuracy compared to high-resolution radiography measurements is only slightly better at $1 / 4^{\circ}$ rotational increment than in the case of $1 / 2^{\circ}$ rotational increment. It should be also noted that the $1 / 4^{\circ}$ increment requires twice as much time for capturing the images as the $1 / 2^{\circ}$ increment.
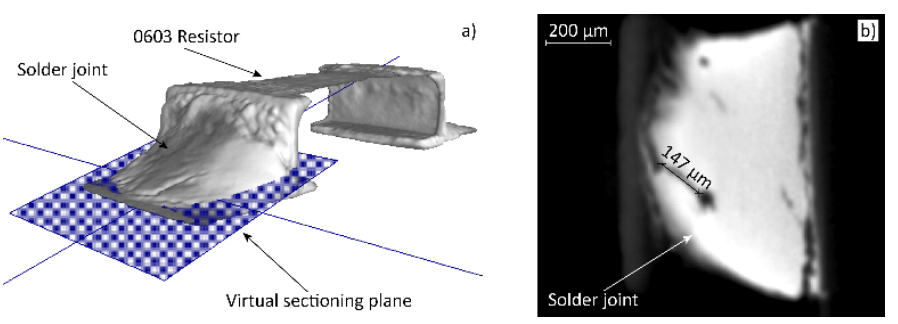

Fig. 4 Investigating a crack inside a SAC solder joint after 2000 TS cycles with CT: (a) defining the virtual sectioning plane, (b) measuring the length in the virtual section which is parallel to the longitudinal axis of the crack (considering the crack as a straight line in a short distance).

Therefore, it can be concluded that $1 / 2^{\circ}$ rotational increment of sample projection is the optimal setting in this investigated case for measuring crack lengths. Nevertheless, the optimal setting of rotational increment depends on the sample dimensions and on the highest magnification. For smaller magnifications - where the voxel (represents a value on a regular grid in three-dimensional space) size is larger - higher rotational increment could provide the acceptable resolution. Therefore, the optimization should be performed for different samples, for different magnifications, utilizing our proposed validation method.

\subsection{Reliability and Quality of Electronic Assemblies}

From the aspect of humidity induced failures (e.g. electrochemical corrosion), the main topics are the investigations of physical, chemical and electrochemical processes, such as the impact of contamination on water layer formation, 
surface finishes and solder alloys to remark a few. The humidity induced reliability investigation of lead-free solders is still an open issue among the researchers, and many studies can be found related to electrochemical corrosion.

In the past year, the BME-ETT department has reached a significant result about the electrochemical corrosion. The corrosion properties of different lead-free micro-alloyed solder alloys were investigated using potentiodynamic polarization tests and scanning electron microscopy with energy dispersive spectroscopy (SEM-EDS) methods. According to the polarization tests, the Ag/Bi-alloyed solder alloy showed the weakest corrosion resistance. The corrosion depth results have confirmed that the silver-bismuth bearing ( $3 \mathrm{wt} \%$ ) lead-free solder alloy have the lowest corrosion resistance compared to the other lead-free ones with no Bi content. The lower corrosion resistance can be explained by relevant silver and bismuth contents and volumes, which were detected in the corroded layers by EDS. The reason could be that the corrosion potential (Ecorr) is shifted towards less noble values with the addition of bismuth and silver [61].

During 2016, BME-ETT joint to the research of Instutue of Ectron Technoology (ITE - Krakow, Poland) about tin pest phenomenon. Tin pest is a spontaneous allotropic transition of $\beta-\mathrm{Sn}$ to $\alpha$-Sn below $13.2^{\circ} \mathrm{C}$. Firstly the transition starts from blemishes, discoloration, mottling. Later these first ones are changed into characteristic warts (Fig. 5, which originates the name "tin pest").

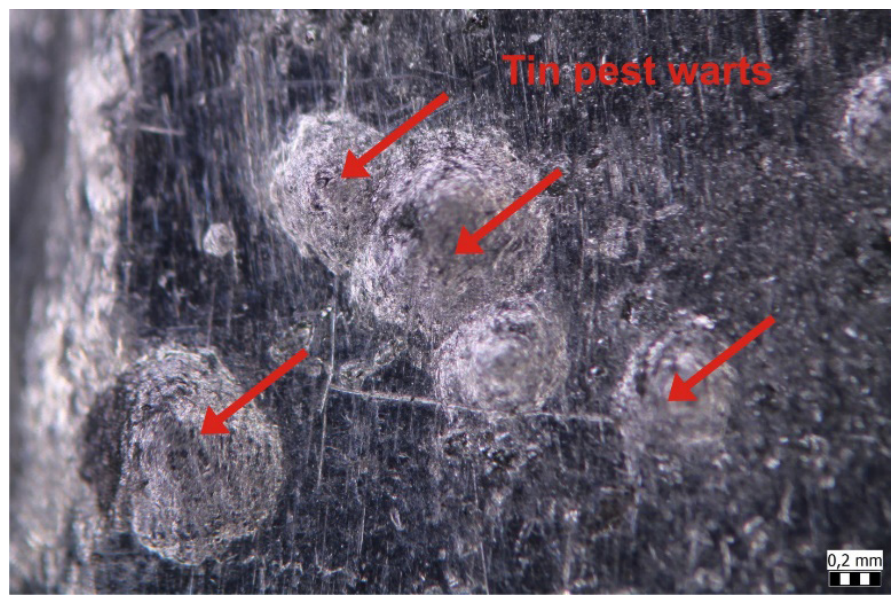

Fig. 5 Image of tin pest warts on the surface of a tin finish

Finally, the sample is changed into the form of the powder leading to the total disintegration of Sn sample. The identification and characterization of tin pest in Sn-rich solders and surface finishes is crucial for electronic devices working in sub-zero temperatures in aeronautical, aerospace and automobile applications.

In this research, the tin pest phenomenon was characterized by electrical resistance measurements. The aim was to compare the ability and sensitivity of the usually applied optical observations by the electrical measurements. It was found that the electrical resistance measurement is a much more sensitive characterization method of tin pest growth than any kind of optical observation [62].

\subsection{Soldering Technology}

Vapor Phase Soldering (VPS) is an emerging reflow soldering method. It is considered as a promising alternative of convection and infrared heating in the electronics industry during soldering. The heat transfer method in the VPS technology is very different from the convection or infrared technologies. During the VPS process, a special heat transfer fluid is evaporated in a closed tank and then the condensation phenomenon is used for heating the assembly.

In the past year, the department has reached significant results in the field of VPS research, the heat transfer and condensate layer formation on the top side of inclined printed circuit boards was investigated by numerical simulations. It was found that even a moderate inclination of the printed circuit board $(>1 \%)$ has considerable effects on the formation of condensate layer and consequently on heat transfer. The thickness differences of the condensate layer can be decreased with an optimized inclination of the printed circuit board and it homogenizes the heat transfer during the process, enabling improved solder joint quality, with reduced overall soldering failure count [63]. During another research, the dependence of heat transfer coefficient during on the substrate properties was investigated by numerical simulations. The results presented dynamic heat transfer coefficient values of the process for different substrate materials. It is shown that the heat transfer coefficient can be characterized with the thermal diffusivity of the substrate material together with the filmwise condensate layer thickness on the substrate. While, the formation of the condensate layer is determined by the thermal diffusivity of the substrate material [64]. The investigations on substrate influence on the heating were extended with measurement approach as well, where the initial results affirm the trends found with the simulation [65].

Thermocouple attachment options were analyzed to improve temperature measurements during the process. It was shown that it is recommended to avoid uninsulated thermocouple wire ends in the vapor zone, as the condensing Galden considerably affects the measurements. The fluid layer of the condensate also increases the uncertainty of polyimide tape attachment method of the thermocouples, while the fluid underflows the tape, which also reduces the quality of adhesion. It was shown that comparing the heating factor of the given temperature profiles yields improved comparison. High temperature solder and Alu-tape attaching methods are suggested for improved measurement repeatability [66].

Vapor Phase Soldering was also considered as a promising alternative of heat transfer on special, heat-sensitive circuit substrates, such as biodegradable boards. [67]

\subsection{Summary}

The research fields of ETT reflect the major trends and results in modern engineering science, which are chiefly engaged with: (i) surface characterization of material properties in the 
nano-level; (ii) application and investigation of electrochemical processes; (iii) novel characterization methods of solders joints microstructure; (iv) reliability problems of electronic assemblies; (v) developments in vapor phase soldering. These topics may serve the interest of the readers of Periodica Polytechnica Electrical Engineering and Computer Science.

\section{Department of Networked Systems and Services}

The Department of Networked Systems and Services is focusing on the key areas of the automated city - one that is predictive and responsive without human intervention. Such a city could avoid traffic congestion before it occurs and distribute resources, such as emergency services and maintenance, without time-consuming human decision-making. Vehicular networks are on the verge of becoming an essential part of our increasingly connected life. However, the evolution of related technologies is still very far from finished: autonomous/automated vehicles, heterogeneous vehicular access environments, large scale deployment scenarios, application and service interoperability, security and privacy still pose serious challenges just to mention a few.

In the following, we present, in somewhat more details, selected research projects from the area of the automated city: (i) V2X communication solutions for connected and autonomous vehicles, (ii) vulnerabilities in connected and autonomous vehicles, (iii) Unmanned Aerial Vehicles in automated cities and (iv) flocking of autonomous vehicles. More information on these and other projects and on our research in general, can be found on the department's web site at http://www.hit.bme.hu.

\subsection{V2X Communication Solutions for Connected and Autonomous Vehicles}

Our department has been deeply involved in international V2X researches since the early days: successful EU projects from the FP6, FP7 and CELTIC programmes employed our expertise in ad hoc radio communication, advanced mobility management, cross-layer optimization, protocol development and performance evaluation. An important milestone and a leap toward industry-motivated research was in 2016 when Commsignia Inc., a leading V2X solution provider company, and BME-HIT took the initiative and formed Europe's first V2X industry-education collaboration - the Commsignia-BME HIT Automotive Communications Research Group, operating within the Laboratory of Multimedia Networks and Services (MEDIANETS).

One of the main research focuses right now is on heterogeneous vehicular networks where the communication diversity covers Wi-Fi, DSRC, CALM, 3G, 4G/LTE/LTE-A, 5G, and Satellite among others. This fact demands the design and implementation of context-aware, resource efficient, fine grained (i.e., flow aware), scalable, and optimized Layer 3 V2X mobility management mechanisms integrated into the
C-ITS standards. To provide the base infrastructure for such mechanisms, we introduced a dynamic network discovery and selection framework relying on the tools of C-ITS standardized Local Dynamic Map (LDM) and Cooperative Awareness Message (CAM) [68]. The proposed solution uses the geographic position and dynamic/static context information of different access networks to predict handover events and proactively prepare for the required mobility management procedures during the V2X communication. The LDM-based predictive decision engine provides prompt and efficient vertical handover (VHO) decisions for V2X applications during different mobility events, while the CAM-supported timely and standard compliant transport of context information provides all the dynamic/static data required for these $\mathrm{VHO}$ decisions. In this way, we can further increase the efficiency of C-ITS services in a standard-compliant manner.

We have proposed a possible architecture for a V2X-integrated traffic light controller systems, which is easy to implement and deploy, uses standardized C-ITS messaging techniques (MAP, SPAT, etc.), and creates an intersection environment for road users and operators where the widest scale of adaptive and dynamic C-ITS applications could be implemented. Our solution was showcased within an interactive demonstration during the Regional Digital Summit 2016 in Budapest, where we tried to highlight the possibilities of highly adaptive and dynamic traffic management systems of Smart Cities strongly relying on V2X technologies.

Motivated by the fact that in-vehicle healthcare applications gain more and more attention, our group also investigates how advanced mobile healthcare (mHealth) services can actively benefit from the advantages of heterogeneous vehicular networks and V2X communication in C-ITS architectures by transferring various, often real-time medical data between drivers/passengers and the designated healthcare infrastructure or medical experts in different scenarios. Our first goal was to propose an in-vehicle healthcare use-case, draft a possible extension of GeoNet (GN) forwarding algorithms and to present comprehensive evaluation of mobile medical multimedia transmission between vehicles over ITS GN GeoBroadcast protocol. Results clearly show that medical multimedia could be transmitted even over $802.11 p$ using GN protocol [69, 70], however, subjective QoE measurements evaluated by medical personnel would also be useful for more precise assessment of medical media quality, and enhanced security/privacy measures must be applied also in the mobility management level of the V2X domain by techniques like [71].

\subsection{Vulnerabilities in Connected and Autonomous Vehicles}

When vehicles are connected to each other and to the Internet, they become potential targets of cyber-attacks. The feasibility of such attacks have recently been demonstrated by 
many researchers: in proof-of-concept attacks, cars have been compromised remotely via wireless interfaces and locally via the OBD interface [72-75]. In case of autonomous vehicles, the danger is even greater, because the physical control of the car is entirely "in the hands" of computers that, as we have seen, can potentially be compromised. So the bright vision of autonomous vehicles can become reality only if we can solve the pressing issue of cyber security; a huge challenge for the research community, as well as for the automotive industry.

In a recent research [76], we showed that it is relatively easy to perform dangerous modifications to the settings of different car equipment (e.g., the airbag in the car) by simply infecting the mechanic's PC or laptop that runs the diagnostic software used to manage those equipment in the car, and replacing the software component responsible for communications between the diagnostic software and the CAN bus with a malicious component that implements man-in-the-middle type attacks (e.g., replay or modification of commands). Indeed, PCs and laptops in mechanics' workshops are not well maintained, often connected to the Internet, and used for various purposes, not only for diagnostics on cars. So, while attacking cars via different wireless interfaces seems to be more exciting than attacking cars via the mechanic's PC or laptop, the latter can in fact be much easier to carry out and more effective in practice.

Current research in our CrySyS Lab is concerned with developing tools and methods for finding vulnerabilities in connected and autonomous vehicles that enable cyber-attacks, as well as developing tools and methods for detecting cyber-attacks and for supporting forensic analysis and incident response. Our aim is to develop an anomaly detection system that analyses CAN traffic and raises alarms when suspicious activities are detected. We also work on privacy issues stemming from massive data collection from vehicles for different purposes.

\subsection{Unmanned Aerial Vehicles in Automated Cities}

At our department, we have been working on UAV research and development for several years, concentrating on the field of radio communication between the UAV and the ground control station and autopilot development. The UAV flight generates several questions, how can they be safe enough to fly above populated areas, where can they fly, can they hold cameras taking pictures about persons, etc. We had suggestions how to create safety UAV aircrafts, safety control algorithms, and safety rules of flight.

One of our main interest is to create a safe autopilot, which can control the UAV not just in easy, but in harsh circumstances too. It means, that the autopilot should be able to control the UAV in stall situation, (or prevent the stall), strange situations, (e.g. inverted flight) and so on. The widely used linear controller based autopilots are not good at this area. The autopilot algorithm basically consists of three main parts, the attitude and state estimator (where we are, is there a bank, going up or down, what is our direction, etc.), guidance (what to do to reach the desired track, e.g.: bank right 20 degree), and the control loops to reach the needed bank.

The first important goal is the precise estimation of the orientation (attitude) of the flying object in the Earth coordinate system. In one of our most important publications [77], we aim to present a novel approach for the design and implementation of an on-board nonlinear state estimation system for an autonomous aerial vehicle. The tasks of such a system include collection of measurement data from different navigation sensors, and estimation of all the quantities describing the system's state of motion based on the system dynamics and the measurement data.

In another paper [78], we have presented a novel approach for the design and implementation of on-board nonlinear control systems for different types of unmanned air vehicles. The essential difficulty of creating such controllers is the inherent nonlinearity of the dynamics of the system, which also introduces a great complexity. Our proposed approach relies on an automatic state dependent coefficient (SDC) factorization procedure, which is capable of handling the dynamic equations of the system on a symbolic level. The resulting linearized system representation is solved by the state dependent Riccati equation (SDRE) method.

Our group is also focusing on the reliable radio connection between the UAV and the ground control station. We developed the communication system of the BHE-MW bxap15 UAV. In 2016, we finished a further development of this radio channel, which became an automatic, adaptive, robust, proprietary telecommunication system with increased range. For Amores Robotics we developed a telemetry radio, which has a tested $15 \mathrm{~km}$ range on a UAV. Now we are working on a multi redundant, failsafe telemetry radio system, which can work at high speed in LOS, lower speed in the case of light hiding, and very low speed via communication satellite in the case of failure of terrestrial communication.

\subsection{Flocking of Autonomous Vehicles}

In autonomous vehicle infrastructures, we are focusing on collective movement of dynamic nodes called "flocking". We conduct research in task allocation, area exploration or target tracking problems where autonomous agents form a flock and can move in a well-defined formation together [79]. However, these agents can be utilized for other tasks as well; dispersing the flock - they do not have to keep a strict formation - enables them to tackle different tasks too. One of these tasks is patrolling in closed or open spaces, such as in a building or on the roads of a city. The challenge of this problem is the coordination of a large number of agents and efficient patrolling on a large graph (such as a large city). Previous solutions focused on centralized approaches: a central entity controlling every agent, constantly telling them where to go in order to visit all the nodes in the graph and minimize interference between the agents. According 
to our latest results, a team of self-organizing agents without any central supervision can solve the patrolling problem efficiently. Agents form partitions on the graph representing the building or city roads to be patrolled, claiming their own territory. This way it is possible to minimize the interference between them and they can patrol their own areas independently. This approach performs well compared to state of the art patrolling algorithms and does not suffer from the prohibitive computational cost that is involved with most centralized approaches. By virtue, this solution scales well and can handle dynamic environments, such as failing agents or added graph nodes, with ease.

Another challenge we are focusing on is the autonomous task allocation, when set of tasks need to be allocated to a set of heterogeneous agents while optimizing overall mission efficiency and reducing expected costs. In recent work, we proposed solutions [80] to solve the aforementioned autonomous task allocation problem, which is an NP-hard combinatorial problem. The proposed algorithm uses distributed actions in order to select the optimal set of agents and does it without any central supervision, relying only on local interactions of the nodes. The algorithm does not depend on the tasks or the requirements directly, since it can select the optimal allocation with respect to the criteria defined by the use case. The result indicate that the proposed algorithm can find the optimal allocation regardless of the network graph of the agents and does it relatively fast compared to the other examined solutions such as random walk, hill climbing, simulated annealing and the full multicast algorithm.

\section{Department of Broadband Infocommunications and Electromagnetic Theory}

The projects and research activities of 2016 at the Department of Broadband Infocommunications and Electromagnetic Theory (HVT) are summarized herein. Our Department is organized in Matrix Scheme, with several cooperating laboratories responsible for specific areas of expertise, such as space technologies, telecommunications covering the frequencies from radio to optical wavelengths, antenna design and characterization, optical and microwave devices, metamaterials and photonic crystals. In-house and commercial codes running on workstations are available, which presents a good starting point for several research and industrial projects. As a unique infrastructure, anechoic chamber and antenna measurement facilities are available at HVT in the range of radio and microwave frequencies.

\subsection{Electromagnetic Simulation and Design Laboratory}

Electromagnetic optimization and inverse problems usually need the solution of many direct problems, which is rather time consuming. This has been inspiring the use of surrogate models that replace the complex direct simulation: they provide approximate results at a much lower computational cost.
The latest developments of surrogate modelling algorithms are related to optimal database generation methods, in order to store pre-calculated direct problem solutions (i.e., training data). A database generation technique using the sparse grid approach is introduced in [81], while a metamodel-based nested sampling strategy is reported in [82].

These results are transferred to industrial applications of non-destructive testing (NdT), among others via the grant of the Hungarian Scientific Research Fund (OTKA, no. K-111987: "Optimization of a magnetic hysteresis measurement-based non-destructive inspection method and its application in materials science", 2015-2018) [83], the János Bolyai Research Scholarship of the Hungarian Academy of Sciences ("Efficient solution and engineering applications of electromagnetic inverse problems", 2016-2019), and the project "Development of inversion procedures based on the exploitation of database of simulated NdT signals" of the French Commisariat á l'énergie atomique et aux énergies alternatives (CEA).

Another activity of long tradition is the numerical simulation of magnetically coupled resonant WPT configurations. The Department is a member of the European COST Action IC1301 "Wireless power transmission for sustainable electronics (WiPE)". Most achievements of the Laboratory are related to the simulation of coupled resonant coils.

The integral equation (IE) formulation developed earlier, which was tailored for thin wire coils of loose winding embedded in homogeneous medium, has been extended for modeling heterogeneous media [84] as well as the dense windings [85]. The IE has been coupled with the finite element method (FEM) [86], and a specific IE formulation based on global series expansion has been developed [87]. All these approaches result in computationally very efficient simulation tools.

The IE modeler has been coupled with a stochastic method that provides the Sobol' indices, by which the sensitivity of the WPT system on the geometry and material parameters can be quantitatively analyzed [88]. Finally, the visualization of power flow has been dealt with [89].

The near-field validation of the numerical models of portable wireless devices is becoming indispensable, especially when checking specific absorption rate (SAR) compliance for regulatory limits by computer simulation. A new method based on comparing measured and computed one-port characteristics of the antennas is proposed, which is much cheaper and faster than the standard validation techniques. Moreover, a theoretical proof is provided for the feasibility of the new method [90].

The radar cross section of small-sized unmanned aerial vehicles can be extremely low, making the perception of such objects impossible by standard radar techniques. However, the typical propeller size of these vehicles gives rise to a resonant behavior at some commonly used radar frequency bands. This phenomenon, a kind of micro-Doppler-effect, is studied by means of anechoic chamber measurements and numerical simulations [91]. 


\subsection{Laboratory of Antennas, EMC and Wave Propagation}

The main profile of the laboratory is the simulation, design, fabrication and measurement of passive and active microwave devices, electromagnetic compatibility (EMC) testing, indoor and outdoor wave propagation simulations and measurements. In a unique way at the BME, the laboratory has a professional anechoic chamber, where the antenna testing and EMC measurements can be performed without any outer interference.

We have investigated the possibility to combine linear and circular polarized antennas for indoor positioning techniques based on signal strength measurement [92]. We have investigated the distribution of the received power by a ray-tracing model and by the measurement of Line of Sight and Non Line of Sight scenarios. Based on the measurements and simulations it can be observed, that it is advantageous of using circularly polarized antennas on the fixed base station and linearly polarized omnidirectional antennas for the mobile devices in indoor environment. The advantage of this solution is that the fluctuations of the received power by the mobile device is reduced as compared to the linearly polarized antennas scenario. In addition, the received power is less sensitive to changes in orientation of the mobile antenna.

The lab has a long-term collaboration with NIVELCO Process Control Co. in the fields of radar antenna design and characterization for tank level radar equipment. Novel planar microstrip antenna is developed with special feeding network and power divider elements for $25 \mathrm{GHz}$ frequency band for replacing ordinary horn and parabolic antennas.

The ESA Project No. 4000114846/15/NL/NDe "Integrated Sentinel-1 PSI and GNSS technical facilities and procedures for determination of 3D surface deformations caused by environmental processes" is a cooperation between Geodetic and Geophysical Institute, Sopron and HVT. The goal of the project is to combine Sentinel IW images processed by integrated Persistent Scatter Interferometry with additional GNSS observations on those important areas, where the use of traditional PSI methods are limited due to complex or unstable ground coverage. Analytical investigations and numerical simulations on corner reflectors were made to determine the limits of truncation. Four scaled (5:1 ratio) corner reflectors (Fig. 6) has been manufactured and measured in anechoic chamber. The measurement data show good agreement with the theoretical results.

\subsection{Laboratory of Multiscale Electromagnetic Systems}

We have conducted research in the topic of metamaterial homogenization [93], imaging application of metamaterials [94, 95] and magnetic hysteresis modelling [96].

Closed form expressions of the Kramers-Kronig and of the multiply subtractive Kramers-Kronig relations are derived to predict the real part of the refractive index from the imaginary part [93]. The accuracy and the convergence rate of the closed form expressions is investigated by calculating the refractive index of a hypothetical double negative metamaterial with predefined parameters. It is shown that the subtractive Kramers-Kronig relation can successfully assist to retrieve the refractive index of metamaterials even when the Kramers-Kronig relation fails. For such scenarios a two-step procedure to extract the real part of the refractive index has been developed. The multilayer fishnet metamaterial serves as example, and the correct refractive index has been retrieved even when the frequency range of the simulations is insufficient or the S-parameters are not accurate enough.

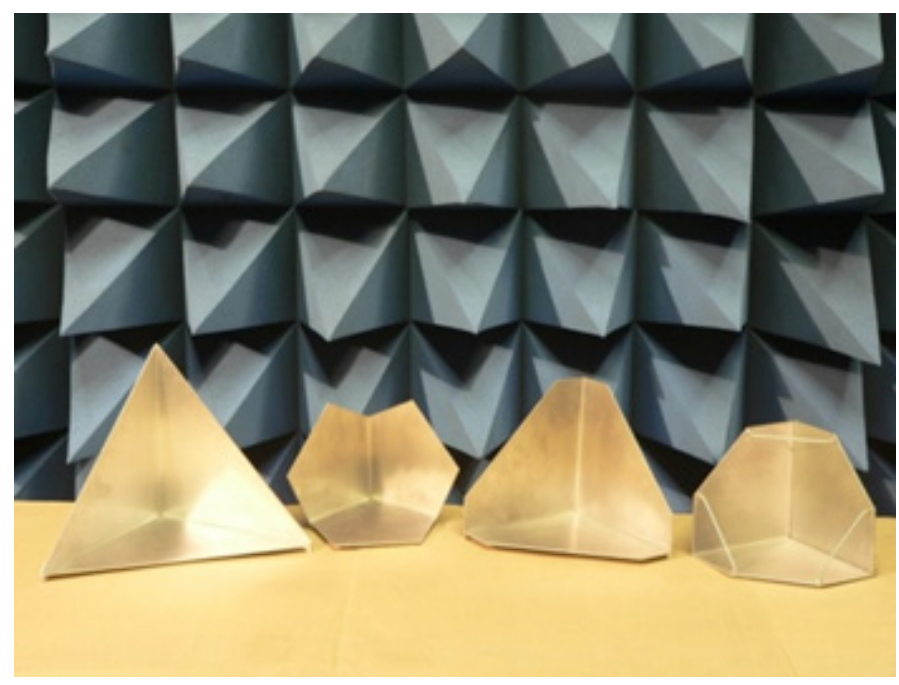

Fig. 6 Scaled corner reflectors in the anechoic chamber

Procedure is developed to construct the image of an extended object placed behind a metamaterial slab as it is seen by an observer, which can greatly differ from the image predicted based on the intensity map [94]. Firstly, the procedure retrieves the dispersion relation of a periodic metamaterial slab from the S-parameters calculated with full wave electromagnetic simulation of the unit cell. Secondly, the procedure utilizes the retrieved dispersion relation in the Transfer Matrix Method to calculate the image of a point source placed behind the metamaterial slab as a function of the observation angle. Knowing the image distance of the point source for all observation angles, the image of an extended object can be efficiently calculated. The procedure is demonstrated with fishnet type metamaterial. Beside its effectiveness, the method has the ability to avoid ghost images and to deal with aberration, which is introduced by the angle dependent properties of metamaterials.

Aberration-free flat lenses are created by introducing phase discontinuities with hyperboloidal distribution on a metasurface [95]. These lenses are able to focus light without aberration under axial illumination but suffer from serious aberration in cases of oblique incidence. Here we introduce a simple design, including an aperture stop and a flat lens with a modified phase distribution that has negligible aberration for incident angles in the range of 
$-40^{\circ}$ to $40^{\circ}$. With this design, diffraction-limited imaging systems can be created without the need for complex lens systems.

A closed form magnetic hysteresis models are developed, which can enable the integration of the hysteresis phenomena in circuit and electromagnetic field calculations [96]. The Preisach function is considered as a product of two special one-dimensional functions, which allows the closed form evaluation of the Everett integral. The deduced closed form expression is included in static and rate dependent hysteresis models and measured hysteresis loops are simulated.

\subsection{Microwave Remote Sensing Laboratory}

The Department joined the international project "Multichannel Passive ISAR Imaging for Military Applications (MAPIS)" that lasts from 2014 to 2017, with the participation of 9 institutes from 5 countries and it is coordinated by the European Defense Agency. The activities of the project are related to passive radars. We conduct research and development activities in the fields of antenna array characterization, adaptive beam forming, spacetime adaptive processing, coverage measurement and simulation of the emitters (e.g., video broadcasting), along with target tracking and ISAR imaging algorithm. Recently developed experimental hardware supports the project: the digital adaptive beam forming is studied via a 4-channel DVB-T band passive radar and the ISAR imaging algorithms are tested with a wide-band active imaging radar. ZDIS algorithms operating in the space, time and space-time domain have been investigated. Special attention is paid to the challenges of small-sized Unmanned Aerial Vehicles (UAVs) detection by combining electromagnetic simulations and anechoic chamber measurements. Consequently, a wide range of activities related to passive radars are in progress [91, $97,98]$ where the cooperation of colleagues with different competences and facilities (electromagnetic modelling, signal processing, hardware development and measurement infrastructure) is essential to succeed in this field.

After the success of Masat-1, the qualification model of Smog-1 satellite is developed entirely with student contributions. Our activities include the development of the electrical power system, on-board computer, communication and spectrum monitoring system, automated and remote controlled satellite control ground stations [99].

\subsection{Optical and Microwave Telecommunication Laboratory}

The research field of this laboratory is specialized in the physical level development and investigation of microwave-photonic components and subsystems, focusing on telecommunication applications. The lab has expertise on wireless and optical communication systems, radio over fiber (RoF) technology, wireless modulation formats, microwave and optical circuit and system design, construction, and test. The recent research activity of the laboratory is relating for optical and microwave fields.
The department has participated in the COST Action IC1101 "Optical Wireless Communications", where the focus of OMT lab was the application of visible light communication (VLC). VLC based indoor localization system was developed and investigated [100]. Hybrid Radio over multimode and free space optic system using mode-filtering techniques was suggested and demonstrated with international partners [101].

Optical access networks related work was supported by Hungarian Scientific Research Fund (OTKA PD 109288 Investigation of in-line and reflective semiconductor optical amplifiers for broadband optical access). It includes simulation and measurement of semiconductor optical amplifier based colorless optical unit for wavelength division multiplexed optical access networks [102].

The lab has long-term cooperation with Ericsson Research in radio over fiber and design of microwave circuit fields. Efficient and optimal optical modulation method for $5 \mathrm{G}$ fronthaul and backhaul systems are important open questions. Distortions and limitation of signal transmission in Coherent-OFDM Systems was investigated theoretically and experimentally [103]. The optical single side band and versatile modulation can be effective solution for the chromatic dispersion limitation. The generation and optimization of it is supported by a Marie Skłodowska-Curie Innovative Training Network, called FIWIN5G: FIber-Wireless Integrated Networks for 5th Generation delivery [104]. The development of novel microwave circuits optimized for RoF applications is a priority research area [105].

Another microwave photonics research field is the millimeter wave and terahertz radio frequency signal generation applying photonics methods. Comparative study of system configurations and investigation of the stability and performance of the generated signal are subjects of heightened interest [106].

A quickly developing research area of the lab is free space optical quantum communication, where our laboratory joined in the Hungarian quantum research group and our members work with photon statistics determination, investigation of laser polarization state and laser sources [107].

\subsection{RF Laboratory for Space Research}

The present activity of the laboratory is focused mainly on millimeter band propagation measurements and modelling under different national and international cooperation. In the TAMOP FIRST project (2012-14) the TV White Space was investigated and a new model was developed for cognitive radio applications [108]. The recent activity of the laboratory is participation in $\mathrm{Ka} / \mathrm{Q}$ band $(20-40 \mathrm{GHz})$ propagation and communication experiments of the ESA (European Space Agency) Alphasat programme. To study the propagation behavior of the Satellite-Earth radio channel a two-band satellite beacon receiver station was developed, built and operated since 2014 [109]. The receiver station in Budapest is part of an international measurement network over Europe. 
Alphasat is also hosting a satellite communications experiment payload to study the characteristics of DVB-S2 satellite transmission on $\mathrm{Q} / \mathrm{V}$ band (Fig. 7). It is a pioneering experiment to study the adaptive modulation system of DVB-S2, the intelligent power control and the diversity receiving opportunities on a $40 \mathrm{GHz}$ satellite Earth radio channel. The participation of our laboratory in the Alphasat programme is founded by European Space Agency.

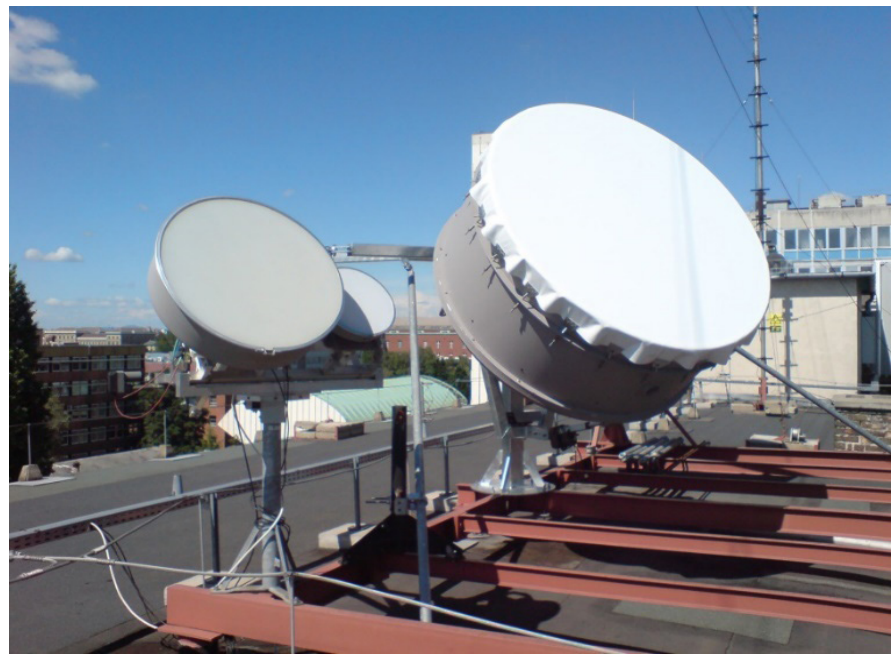

Fig. 7 The Alphasat receiver antennas at BME-HVT

\section{Department of Control Engineering and Information Technology}

As for the summary of our results achieved during the previous years, subsections present specific contribution to our major research fields. For further details, the reader may refer to the proceedings of the workshop [110] specifically organized to collect the latest results in our fields of competences.

\subsection{Geometric Modeling}

We investigated multi-sided surface modeling for interpolating general topology free-form curve networks, and have formulated a new surface type that generalizes the well-known tensor-product Bézier patches [111]. We continued our research in digital shape reconstruction, in particular, how to detect and enforce various geometric constraints for perfecting reconstructed CAD models [112].

\subsection{Dynamic Positron Emission Tomography}

In Dynamic Positron Emission Tomography, the spatio-temporal distribution of radioactive isotopes should be reconstructed from observed simultaneous photon hits that are born as by-products of the decay. Due to the poor statistical determination of the measured data, we have to apply regularization both in the 3D spatial and $1 \mathrm{D}$ temporal domains. We have investigated the possibilities for such $3+1 \mathrm{D}$ regularization [113].

Inverse problems are solved by iterating a physical simulation of the measurement of the current state estimate, comparing the simulated result with the measured data, and finally updating the state estimate. Thus, the efficient physical simulation of particle transfer in heterogeneous media is essential. We have developed a behaviorally valid model that works differently than the real particles, but provides the same expectations and its relevant parts can analytically be evaluated [114].

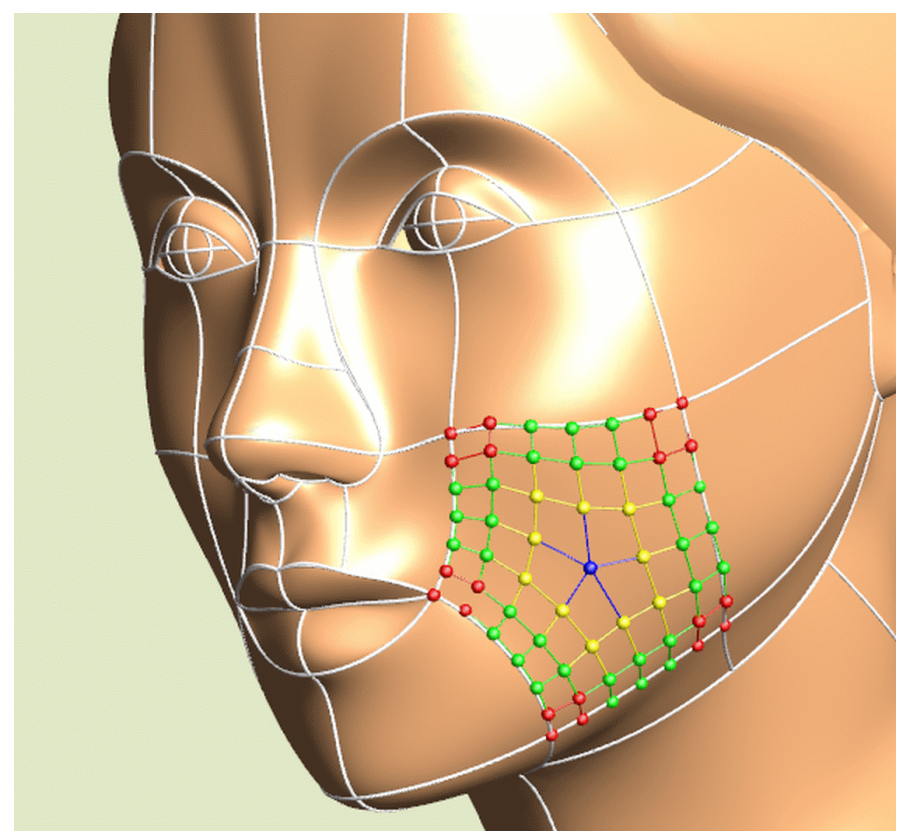

Fig. 8 Control structure of the new patch model

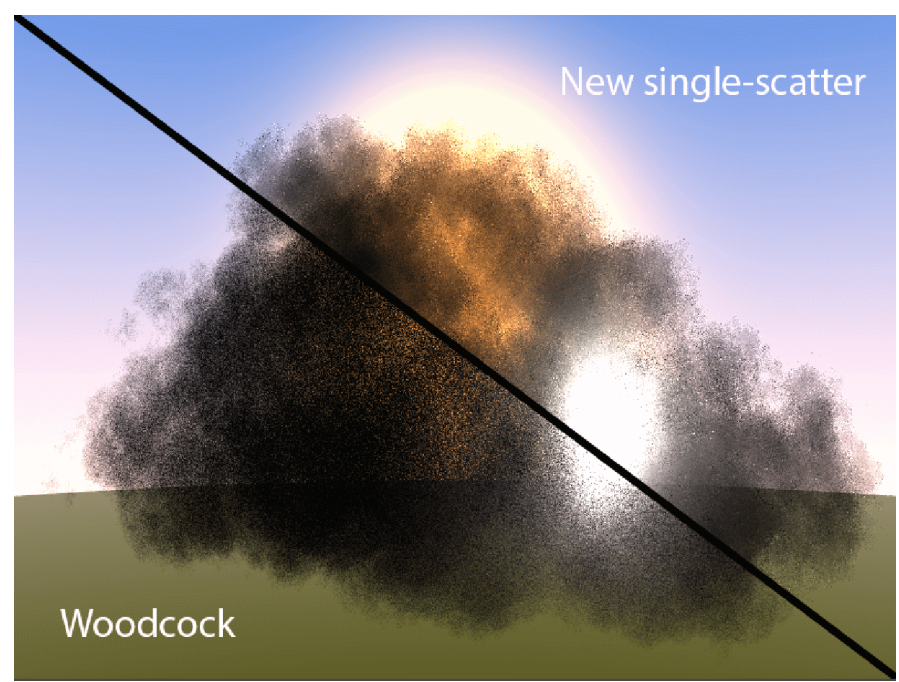

Fig. 9 Comparsion of classical Woodcock tracking and the proposed behaviorally valid model in single scattering simulation.

In volume visualization and tomographic reconstruction, box splines are mainly used as reconstruction kernels. The usual way of tailoring a box spline to a specific lattice is to span the box spline in the principal directions of the lattice. We have shown that this amplifies the anisotropic effects, and proposed an isotropic retailoring of box splines to lattices [115].

We developed a method that detects anatomy regions in three-dimensional medical images [116]. Each axial slice of the image is labeled according to the anatomy region it belongs to. 
The core of the algorithm is based on a two-dimensional feature extraction that is followed by a random-forest classification.

\subsection{Software Quality}

Within the domain of software quality, we focus on multimodel approaches. We use software quality approaches in an integrated way considering a basic set of quality characteristics. We also research the ways of enhancing existing testing methodologies based on the characteristics of the software.

We continued working on the development of a new methodology based on text analyses and data mining via performing similarity analyses between international quality approaches. Here, the target was to develop a methodology that enables auditors and other stakeholders to perform automatic and quantitative analysis at software developing organizations in comparison to a chosen international quality approach. Such analysis and comparison require lots of engineering work by experts, therefore resulting in inefficient human resource utilization. We developed a new methodology, called KA-TA, which can be used for the automatic and quantitative analysis as part of an assessment process to identify strengths and weaknesses and create similarity maps. This methodology can also be the basis of a set of quantitative metrics to measure the process conformance level of an organization. Our activity was also connected to testing and quality issues in blood sugar monitoring systems, including the definition of continuous integration criteria, as well as real time monitoring of the measurement data. Integrating Jbehave with the monitoring framework is a further goal.

Both development and testing methodologies were improved in a telecommunication company based on our research results $[117,118]$.

\subsection{Biomedical Engineering Lab}

On the field of Model Based Therapeutics, significant results are achieved with cooperation of several international institutions including University of Canterbury, New Zealand. This research was part of the eTime - Engineering Technology-based Innovation in Medicine project supported by the EU FP7 Marie Curie IRSES program (Grant No. 318943) [119].

The previous theoretical results are already introduced into the regular medical care in form of an advisory system, STAR. Now a retrospective analysis is published proving the safety and efficacy of the therapy, as well as showing similarities and differences between patient groups [120].

The methodology has been further improved by introducing novel control strategy [121] and applying stochastic differential equation and suggesting novel methods for identification insulin sensitivity of the patients $[122,123]$ that result in promising improvement in the treatment outcome.

Similar model based approach is applied for solving clinical problems during mechanical ventilation [124]. The therapy is currently under validation by a major clinical trial [125].

\subsection{D Shape Recognition for Adaptive Tangible Augmented Reality}

Some interfaces combine the idea of Tangible User Interfaces (TUIs) with Augmented Reality (AR), creating an environment in which users touch real-world objects, while interacting with virtual ones. This allows for intuitive user interfaces, while removing a conflict between different senses. In most cases, however, Tangible Augmented Reality (TAR) systems require the preparation of the physical environment to function (markers, or special real-world objects).

Our aim is to create a TAR system that can process a previously unknown environment and find real-world objects that are fitting placeholders for the virtual objects the application requires. Our goal is to ensure that virtual objects are assigned to real ones that are similar in shape. In our system, the shape of an object or a scene is represented as a graph of primitive shapes. The key algorithm of our system is a learning classification algorithm that uses 3D shape data to pair virtual and real objects. Our system uses this algorithm to find a subgraph that belongs to a fitting object in a 3D scene.

In our research [126, 127], we use node-by-node graph classification to improve accuracy, and to allow for easy alignment of the virtual objects. For this purpose, we proposed a novel method for embedding the local context of graph nodes into vector spaces, which was found to improve the quality of classification. To reduce the dimensionality of the graph feature vector, we also proposed a discriminant analysis method that handles between class discrimination and the separation of nodes within a single graph at the same time.

\subsection{System Level Synthesis Tool for Designing Task- dependent Multiprocessing Systems}

Multiprocessing can be considered the most characteristic common property of complex digital systems. Due to the more and more complex tasks to be solved while fulfilling often conflicting requirements (cost, speed, energy and communication efficiency, pipelining, parallelism, the number of component processors, etc.), the system architecture is strongly determined by the task to be solved. The rapid development in this field demands new system-level synthesis methods supporting the designer in finding, optimizing and evaluating the proper solutions and eliminating the intuitive steps in the synthesis procedure as far as possible. In this research project, an experimental system level synthesis tool (called DECHLS) has been developed relying on decomposition and high-level synthesis algorithms. The tool DECHLS receives the to be solved written in programming language $\mathrm{C}$ and after performing the task decomposition assigns the subtasks into component processors considering the requirements and their priority order definable by the designer.

Our current research aims at finding decomposition algorithms that are better suited to DECHLS and at developing 
more effective methods for handling loops and arrays in the task description. The application of the DECHLS tool has been illustrated and its various algorithms have been compared and evaluated on practical examples [128, 129].

\subsection{Robotics and Control Engineering}

Research activities aimed to extend the power of modelling, state estimation and feedback design techniques in the domain of mechatronic systems considering especially underactuated and car-like dynamics. A modelling approach reusing techniques from robotics has been successfully applied for realistic car dynamics (including 21 DOFs, c.f. Fig. 10) and to design a hierarchical control architecture [130].

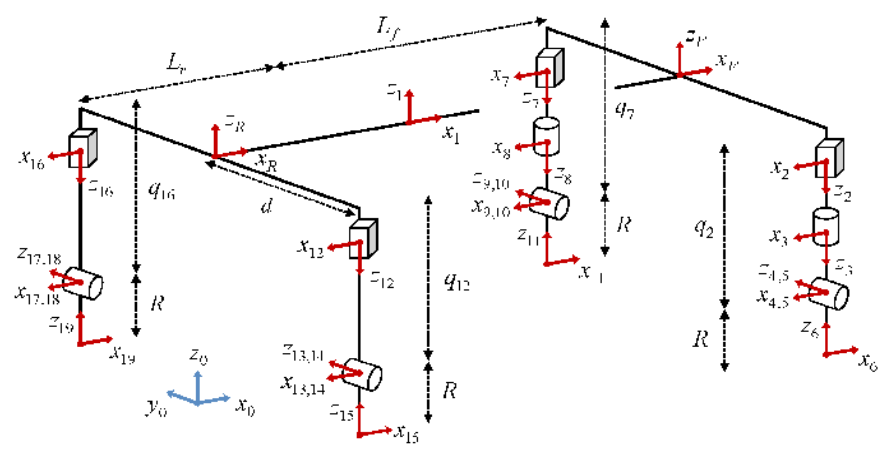

Fig. 10 Degrees of Freedom of a full vehicle model

A sensor fusion technique is reported for the cooperative localization of multiple mobile robots in indoor environment [131]. Results show that cooperative localization provides more accurate estimates than the precision of any of the sensors of individual robots.

For underactuated systems subject to oscillatory behaviour and frictions impeding its detection, a UKF based technique is proposed to estimate the oscillation using a novel adaptive scaling method [132]. The method is successfully applied to crane systems where a new sensing idea is also introduced using laser slot sensors as depicted in Fig. 11.

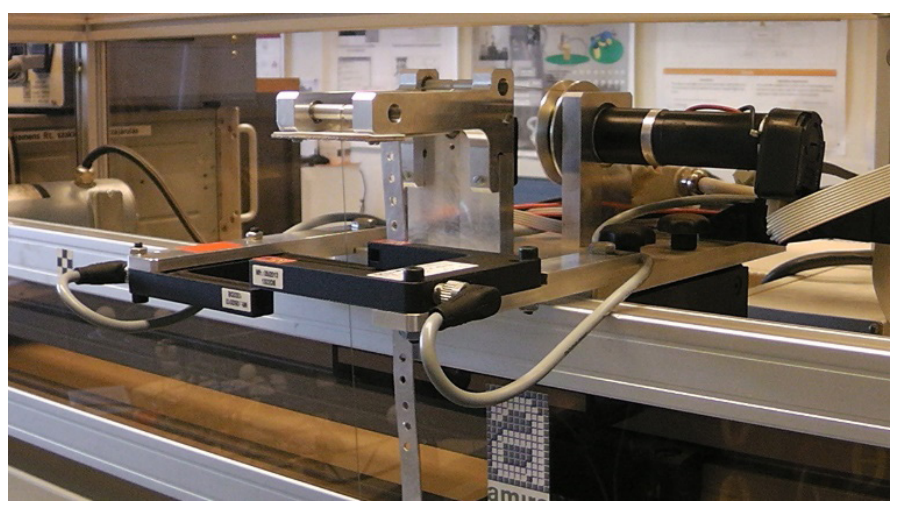

Fig. 11 Experimental crane system for the study of underactuated mechanisms

\section{Department of Measurement and Information Systems}

The focus of the research, development and education activities of the Department is Cyber-Physical Systems (CPSs) with intelligent and critical applications. CPSs turn embedded systems into smart objects by tightly integrating computation, communication and signal processing approaches with physical processes. Our activities include foundational research to provide core insights and algorithms, as well as applied research to develop or innovatively exploit existing technologies in several application domains.

Our recent results contribute to different aspects of CPS:

- Core technologies at smart interconnect and data-to-information levels: Real-time networking, digital signal processing, energy-efficient high-performance computing.

- Design and analysis at CPS level: Modelling techniques for CPS, verification approaches for critical systems, monitoring of dynamic and adaptable CPS.

- Technologies at cognition level: Ambient intelligence, combination of data and knowledge.

- Specific applications: Home health monitoring, medical imaging, reconfigurable robotic systems.

These results are summarized in the next sections.

\subsection{Real-Time Network Embedded Systems}

In this field our research and development covers autonomous systems (including autonomous vehicles), IoT, IIoT, Industry 4.0, and CPS, with special emphasis on safety-critical and/ or real-time embedded applications. Our practical experience includes microcontroller based and application processors based embedded system design, embedded real-time operating systems including Linux [136] and FreeRTOS, embedded platform virtualization, sensor fusion and actuators [137], and application/ algorithm development. Besides classic protocols, we focus on clock and frequency synchronization in Time Sensitive Networks (TSN) [138], and web technologies, including the modern IoT application layer protocols, such as REST, CoAP or MQTT.

We performed research and development of real-time communication technologies such as TTEthernet, Audio Video Bridging, and the new TSN standard, and time synchronization technologies such as IEEE 1588 and 802.1as (Fig. 12). Our development in these fields targeted end device and network device firmware, and real-time applications. We are also active in the automotive and vehicular communication field by using CAN, LIN, and FlexRay, automotive Ethernet, and HIL testing.

\subsection{Digital Signal Processing}

We continued the long-term research in Analog-to-Digital Converter testing, with new results on the achievable precision of the estimated parameters of a sine-wave fitted on quantized samples [141-143]. Such research allows the improvement of the ADC test procedures defined in IEEE-STD-1241. 
We were also active in acoustic signal processing, developing a new least squares procedure for optimization problems [144].

Recently the technological development allowed measurement data transfer in unreliable systems like sensor networks, where data loss is inevitable. The spreading of the idea of Internet of Things has amplified the problem, which motivated new research. The actual results are about FFT-based spectrum estimation in the case of data loss $[139,140]$.

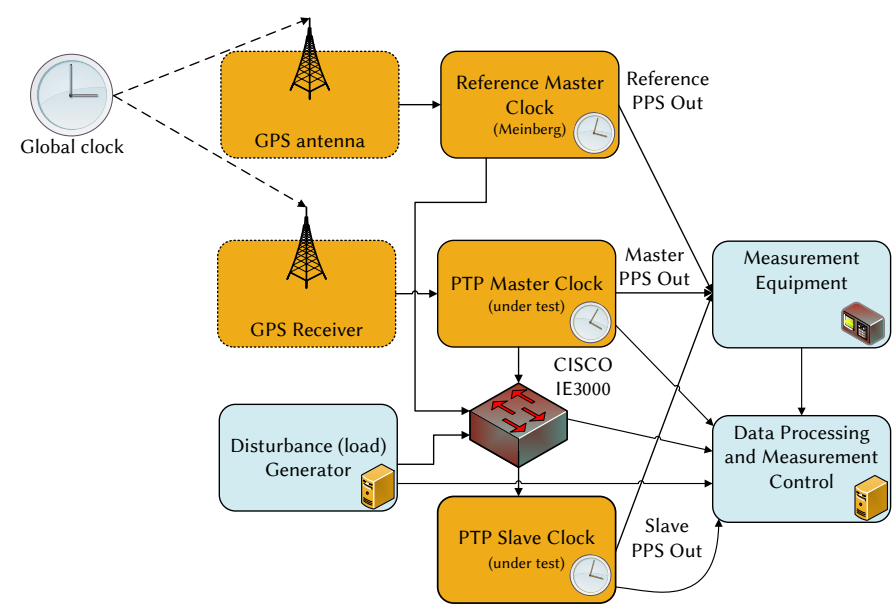

Fig. 12 Time synchronization test system of BME MIT

\subsection{Energy-Efficient High-Performance Computing}

In data- or communication-intensive applications, traditional multi-core CPU architectures achieve only moderate computational performance while consuming a lot of energy. We focus on application specific accelerators realized by FPGAs or GPU based computational units, since this way more efficient heterogeneous systems can be realized both in the extreme data-center like environments and in performance demanding embedded applications. The design methodology for heterogeneous systems is based on the data-flow paradigm and supported by unified software development environments.

The embedded heterogeneous computing architectures that we develop can be used efficiently in various applications, e.g., real time video processing systems used in autonomous driver assistant systems (ADAS), object detection and collision avoidance; high-precision data acquisition systems; communication packet filtering and classification. When used in supercomputing centers for large data set simulations, our approach to heterogeneous computing systems offers one or two order of magnitude better performance/power ratio than the traditional homogeneous systems.

\subsection{Modelling Techniques for CPS}

Large-scale system development efforts require the collaboration of several engineers. Therefore, modelling tools need to provide secure views of the model repository that contain filtered and/or obfuscated set of artifacts. We developed a collaborative modelling framework with the distinguishing features of fine-grained, rule-based model-level access control. The synchronization between views is achieved by incremental and bidirectional model transformations [153].

Synthesizing prototypical contexts is often needed for testing context-dependent behavior of autonomous CPS and for validating CPS modelling tool-chains. However, the synthesis of scalable, consistent, diverse and realistic models is a major foundational research challenge. We proposed an iterative process for generating consistent context models by calling existing logic solvers using various approximations of metamodels and constraints to improve overall scalability [161].

\subsection{Verification Approaches for Critical Systems}

Due to the heterogeneity of CPS, there is no single technique that can verify all of their aspects (e.g., hardware, software, communication). Our research aims to integrate different, abstraction-based techniques into a common, configurable verification framework, enabling to select the most appropriate analysis method for each aspect [157].

In case of concurrent systems, the phenomenon of state space explosion often prevents the successful formal verification of the specified properties. We proposed a new on-the-fly, incremental Linear Temporal Logic model checking algorithm that scales well with the size of models [160].

Safety-critical industrial systems employ dedicated hardware, especially safety PLCs with special programming languages. We contributed to the development of a formal verification method that was validated at CERN [154].

Answering reliability, safety and performability analysis questions for dynamic CPS can often be reduced to the solution of stochastic models. We designed a stochastic analysis framework that supports different symbolic and numerical algorithms to compute reward and engineering measures on models of CPSs [159].

\subsection{Monitoring of Dynamic and Adaptable CPS}

CPSs typically have context-dependent (often autonomous) behavior and complex interactions with their environment, which makes the analysis of such systems difficult. To ensure the safe operation of CPS, we developed run-time monitoring techniques for verifying both high-level safety properties and assumptions about the context [156].

Many distributed smart CPS implement the control logic over a stream of events, which may need extreme scalability in a distributed environment with a massive number of nodes [158]. Complex event processing (CEP) offers well-founded techniques to capture critical event sequences observed on the event streams within a given time window. We developed a CEP engine tightly integrated into the open source VIATRA reactive and incremental transformation framework [155, 162]. 


\subsection{Ambient Intelligence}

The focus of our research and development in the area of ambient intelligence was on the Active Assisted Living. To support the quality of the independent personal life at home, both the physiological (e.g., diseases, accidents) and psychological aspects (e.g., cognitive decline) are dealt with.

Data collection and intelligent processing are the most basic functions in an AAL system, which can be implemented with sensors arranged in a network (Fig. 13). In this area, legal and ethical problems should be taken into account as well, and maximal privacy and minimal disturbance of the person should be maintained. Therefore, we apply a camera-less approach and simple sensors (e.g. motion and contact sensors). Inference from the low-level information is a challenge for the intelligent processing and inference system.
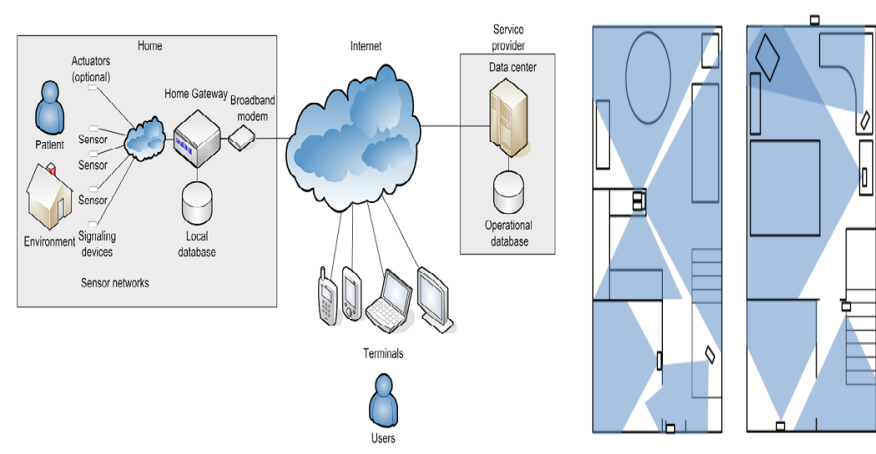

Fig. 13 AAL architecture and a room monitored using motion sensors

\subsection{Combination of Data and Knowledge}

New molecular measurement technologies, particularly in genetics, fundamentally changed the way of biomedical research by the generation of massive amount of data. The slow clinical utilization of these information sources led to the realization that further development depends at least as much on the fusion of data and knowledge, as on further technological breakthroughs in data generation. Additionally, the focus has been shifting towards the collection of real-life data in very large populations, covering information about life style, environment and general health state, including detailed information about morbidities and drug usage.

This collection is supported by wearable electronic devices and other supportive technologies of ambient assisted living. This novel "big health data" provides an opportunity for a systems-based investigation of genetic, personal, environmental and societal aspects of health, ageing and diseases. We developed new methodologies for finding biomarkers of complex diseases [149], for predicting new targets of drug candidates [150], and applied our Bayesian systems-based multilevel analysis in multiple biomedical domains [151, 152].

\subsection{Home Health Monitoring}

Non-invasive blood pressure measurement may result in incorrect values especially for patients with cardiovascular diseases. We suggested considering the occlusion with the cuff as excitation and evaluating the response of the cardiovascular system, using the change in pulse wave transit time as extra information [135].

Sitting in a Huple, a patented hemisphere-like tool, permanently stimulates the vestibular system thus improving the balancing ability of children with movement disorders. Our idea is attaching $\mathrm{x}$-IMU, a wireless $3 \mathrm{D}$ orientation sensor, to the Huple, thus it can serve as an input peripheral for simple PC games. Children are thus motivated to perform balance training and participate in the game-like test. This improves the accuracy and reproducibility of the assessment [133, 134].

\subsection{Medical Imaging - Digital Tomosynthesis}

We contributed to the development of a whole lung diagnostic system using digital tomosynthesis (DTS, Fig. 14) [147, 148]. In a DTS system, nodule detection is based on 2D image processing on the separated slice images (that are reconstructed from projections), and a joint analysis of the $2 \mathrm{D}$ results. We proposed efficient, domain-specific filters for the enhancement and classification of bright, rounded structures. In addition, we developed algorithms to reduce false positive findings that come from nodule-like vessel shadows in the images. The system was evaluated on the first DTS scans. In a database of $\sim 2000$ nodule candidates, $97 \%$ of nodules could be detected, while producing on average 31 false positives per scan.

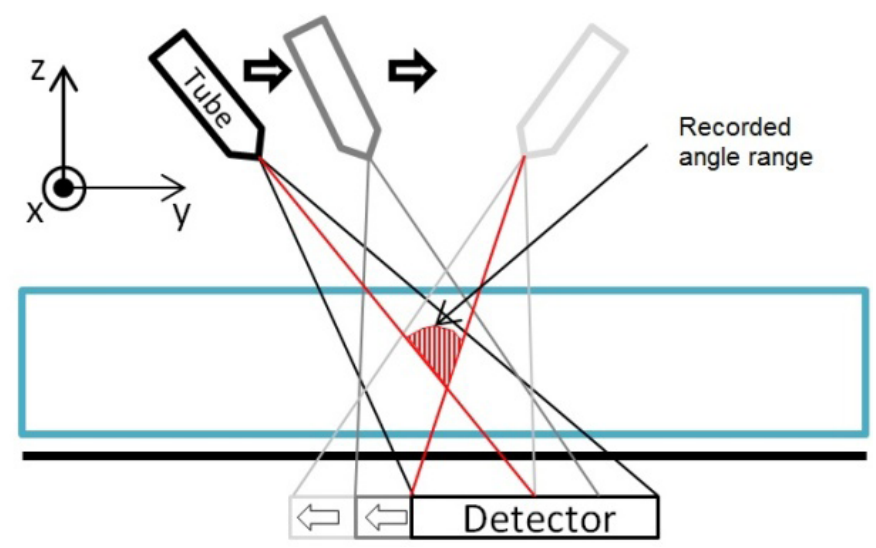

Fig. 14 Recording arrangement for the linear tomosynthesis

\subsection{Reconfigurable Robotic Systems}

Configurability is one of the essential abilities of the modern industrial, service or logistic robotic systems. It may range from a change of parameters, through re-programming the robots, up to being able to alter the physical structure of the system (especially its sensors and actuators).

We developed models and algorithms to support (re)configuration [145], utilizing our experience in knowledge representation. The configuration ability can be offered to the user, or performed by the robot itself. Unskilled users need an easy-touse natural interface to interact with the robotic system. Our research was focused on modelling robots as cooperating and/ 
or conflicting intelligent agents in a mixed-initiative human-robot task environment. We provided the operator with a flexible natural language interface [146] (Fig. 15), coupled with multi-agent infrastructure, dynamically composed and reconfigured according to the tasks and skills of the robotic system. The multi-agent infrastructure was fused with the ROS robotic middleware to provide communication.

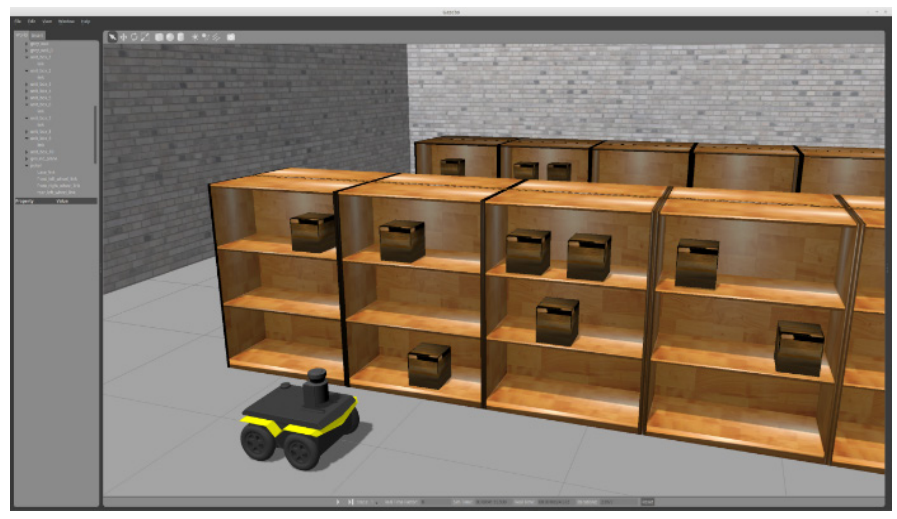

Fig. 15 Voice-controlled robot in a warehouse (ROS Gazebo simulation)

\subsection{Summary}

The previous sections demonstrated the key research results of the Embedded Systems, Intelligent Systems, and Fault Tolerant Systems research groups of the Department. The research activities focused on different aspects of CPS that reflect the major trends in science and engineering, stipulating both educational and professional activities.

\section{Department of Computer Science and Information Theory}

The members of our department perform research in several areas of mathematics and computer science, including combinatorics, theory of graphs, hypergraphs and matroids, algorithms and data structures, computational complexity, stochastic processes, information theory, data science etc. Special emphasis is given to the applications of these tools in various areas of engineering as well.

In the present survey, we concentrate on two selected topics only. First, on a more practical one on sports elimination and on a more theoretical one on combinatorial number theory.

\subsection{The Sports Elimination Problem from a Computational View}

Consider an on-going sports competition between a set of teams, where each participating team has currently a certain score and still some matches to play. Can our favorite team become a winner of the season? More precisely, given the current scores and the set of remaining matches, is it possible that these matches end in such a way that our team will finish with the maximum score among all teams? This question occupies not only players, coaches and managers of teams, but also many sports fans, and has lately attracted attention from numerous mathematicians and computer scientists as well. In our paper [165], we studied the detailed computational complexity of this problem.

To express our problem in a general way that can model competitions for different kind of sports, we let $S$ denote the set of possible outcomes for a match; e.g., $S=\{(0,3),(1,1),(3,0)\}$ for the European football in the FIFA scoring system: the winner obtains 3 points, the loser gets 0 , and a draw yields 1 points for each team. Then the question of whether a given team can still win the competition at a certain moment is called the Generalized Sports Elimination $(S)$ problem, or GSE $(S)$.

Kern and Paulusma [173] characterized those sets $S$ of outcomes for which $\operatorname{GSE}(S)$ is computationally tractable: namely, $\mathrm{GSE}(S)$ can be solved in polynomial time if $S=\{(i, K-i): 0 \leq i \leq K\}$ for some integer $K$ (we call such sets $S$ complete), and it is NP-complete in all other cases.

In order to refine the computational complexity of $\operatorname{GSE}(S)$ for non-complete sets $S$, we rephrased it as a graph labelling problem with vertex capacities where vertices represent teams, arcs represent the remaining matches of the competition, and the task is to allocate a label from $S$ to each arc in a way that each vertex obtains a score (determined by the labels of its incident arcs) that does not exceed its capacity.

Our algorithmic approach was to identify computationally easy special cases, and to identify certain properties of an input that might lead to efficient algorithms for $\operatorname{GSE}(\underline{\mathrm{S}})$.

We applied the framework of parameterized complexity [170], whose basic idea is to associate with each input $I$ of a given problem an integer $k$ called the parameter, and examine the running time of a given algorithm as the function of both the input length $|I|$ and the parameter $k$. The aim is to obtain an algorithm that runs in time $f(k)|I|^{\mathrm{O}(1)}$ for some computable function $\mathrm{f}$; such an algorithm is called fixed-parameter tractable (FPT). Though the running time can depend exponentially on the parameter, its dependence on $|I|$ is only a polynomial of constant degree.

We first identified certain conditions that render $\operatorname{GSE}(S)$ polynomial-time solvable. Namely, we proved tractability of the case when the (undirected version of the) input graph is a forest, by proposing a simple linear-time dynamic programming algorithm. We also asserted polynomial-time solvability of the cases when the input graph is a cycle or a graph with maximum degree 2. The latter result extends the work of Bernholt et al. [164] (dealing with the European football elimination problem) and is in sharp contrast to the fact that $\operatorname{GSE}(S)$ remains NP-complete even for graphs with maximum degree 3.

After identifying these easy cases, we performed a thorough investigation on how certain parameters of the input graph modify the tractability of the problem, and how small deviations from the tractable cases can be handled. To this end, we considered various parameters of the input graph, which aim to measure its 
distance from a certain class of tractable instances. Namely, we focus on three types of parameters of the input graph:

- degree parameters such as the number of vertices with degree more than two;

- feedback parameters, describing how many vertices or edges must be deleted from the input graph in order to obtain a forest;

- width parameters such as treewidth and pathwidth, which measure the 'tree- likeness' of the input graph in a more elaborate way.

For degree parameters, we were able to provide efficient FPT algorithms. Interestingly, the tractability of $\operatorname{GSE}(S)$ with respect to feedback parameters differs greatly depending on whether we deal with the size of a mini-mum feedback edge set (in which case we proposed an FPT algorithm), or with the size of a minimum feedback vertex set (yielding intracta-bility). Regarding width parameters, we obtained that for each fixed integer $w, \operatorname{GSE}(S)$ becomes polynomial-time solvable on graphs with treewidth at most $w$. However, this result cannot be strengthened to achieve fixed-parameter tractability, since we proved W[1]-hardness with respect to the parameter $w$. Furthermore, $\operatorname{GSE}(S)$ proved to be $\mathrm{W}[1]$-hard when parameterized by the pathwidth of the input graph.

In a subsequent article [166], we also investigated the complexity of the following generalization of $\operatorname{GSE}(S)$ : given the current situation in an on-going sports competition, we ask whether it is possible for our distinguished team $T$ to obtain a final standing with at most $r$ teams finishing before $T$. We studied the computational complexity of this problem, addressing it both from the viewpoint of parameterized complexity and of approximation. We focused on a special case equivalent to finding a maximal induced subgraph of a given graph $G$ that admits an orientation where the in-degree of each vertex is upper-bounded by a given function. We obtained a $\Theta(\log$ $|V(G)|)$ approximation for this problem based on an asymptotically optimal approximation we present for a certain matroid problem in which we need to cover a base of a matroid by picking elements from a set family.

\subsection{Combinatorial Results}

In his influential papers Roth has shown that if a subset $A$ of $\{1,2, \ldots, n\}$ does not contain a three-term arithmetic progression, then $|A|=O(n / \log \log n)$ as $n$ grows. Since then, estimating the largest possible size of such a set has become one of the central problems in additive combinatorics. Roth's original results were improved by Heath-Brown, Szemerédi, Bourgain, Sanders, and Bloom, the current record due to Bloom being $|A|=O\left(n(\log \log n)^{4} / \log n\right)$.

It can be easily seen that Roth's problem is essentially equivalent to estimating the largest possible size of a subset of the cyclic group $Z_{n}$, free of three-term arithmetic progressions.
This makes it natural to investigate other finite abelian groups.

We say that a subset $A$ of an (additively written) abelian group $G$ is progression-free, if there do not exist pairwise distinct $a, b, c$ elements of $A$ with $a+b=2 c$, and denote by $r_{3}(G)$ the largest size of a progression-free subset $A$ of $G$. For abelian groups $G$ of odd order, Brown and Buhler and independently Frankl, Graham, and Rödl proved that $r_{3}(G)=o(|G|)$ as $|G|$ grows. Meshulam [12], following the general lines of Roth's argument, has shown that if $G$ is an abelian group of odd order, then $r_{3}(G) \leq 2|G| / \operatorname{rk}(G)$ (where we use the standard notation $\operatorname{rk}(G)$ for the rank of $G)$. Despite many efforts, no further progress was made for over 15 years, till Bateman and Katz [163] in their ground-breaking paper proved that $r_{3}\left(Z_{3}{ }^{n}\right)=\mathrm{O}\left(3^{n}\right)$ $n^{1+\varepsilon}$ ) with an absolute constant $\varepsilon>0$.

Abelian groups of even order were first considered by Lev in 2004, who, as a further elaboration on the Roth-Meshulam proof, has shown that $r_{3}(G)<2|G| / \operatorname{rk}(2 G)$ for any finite abelian group $G$; here $2 G=\{2 g$ : $g$ is in $G\}$. For the homocyclic groups of exponent 4 this result was improved by Sanders, who proved that $r_{3}\left(Z_{4}^{n}\right)=\mathrm{O}\left(4^{n} / n(\log n)^{\varepsilon}\right)$ with an absolute constant $\varepsilon>0$. Croot, Lev and Pach [169] further improved Sanders's result. They showed that $r_{3}\left(Z_{4}{ }^{n}\right)=\mathrm{O}\left(3.62^{n}\right)$.

We note that the exponential reduction in this theorem is the first of its kind for problems of this sort. Starting from Roth, the standard way to obtain quantitative estimates for $r_{3}(G)$ involves a combination of the Fourier analysis and the density increment technique. In contrast, in [169] a new variant of the polynomial method is developed and used without resorting to the familiar Fourier analysis-density increment strategy.

Although this paper was just published on the arXiv in May 2016, the method already has a large number of interesting applications. Now we briefly mention some of them.

- Ellenberg and Gijswijt [171] by a modification of the argument managed to give an exponentially small upper bound for $\mathrm{r} 3(\mathrm{Z3n})$, which question is known as the "cap set problem". Namely, they proved that $\mathrm{r} 3(\mathrm{Z3n})=\mathrm{O}(2.756 \mathrm{n})$.

- Tao on his blog presented a "symmetric" formulation of this method and Petrov gave a third formulation with group rings.

- As a corollary of the cap set result the Erdős-Szemerédi sunflower also got proven, the best bound is obtained by Naslund and Sawin with the help of this new variant of the polynomial method.

- Kleinberg, Sawin and Speyer determined the growth rate of tri-colored sumfree sets.

- Blasiak, Church, Cohn, Grochow, Naslund, Sawin and Umans ruled out one possible approach to improving the exponent of matrix multiplication, namely using the simultaneous triple product property (STPP) for abelian groups of bounded exponent

- Fox and Lovász gave a tight bound for Green's arithmetic triangle removal lemma. 
- Green proved an analogue of Sárközy's theorem in function fields.

In earlier papers [175-177] Pach studied several questions arising in combinatorial number theory, where combinatorial lemmas play an important role. Recently, as a continuation, in [178] Pach and Sándor investigated how small the density of a multiplicative basis of order $h$ can be. While the additive basis is a popular topic in additive number theory, much less attention was devoted to the multiplicative basis. A subset $B$ of $\{1,2, \ldots, n\}$ is called a multiplicative basis of order $h$, if every element of $\{1,2, \ldots, n\}$ can be written as a product of at most $h$ elements of $B$. Let $G_{h}(n)$ be the size of the smallest multiplicative basis for $\{1,2, \ldots, n\}$. The authors give an asymptotically precise formula by showing that $G_{h}(n) \sim \pi(n)$, where $\pi$ is the prime counting function. Moreover, the error term $G_{h}(n)-\pi(n)$ is also determined up to a constant factor (not depending either on $h$ or on $n$ ), namely, it is proved that $\pi(n)+0.5 h n^{2 /(h+1)} / \log ^{2}(n)<G_{h}(n)-\pi(n)<\pi(n)+151 h n^{2 /(h+1)} / \log ^{2}(n)$.

Several other estimations are given in the infinite case, as well, where the asymptotic and logarithmic density of a multiplicative basis of order $h$ for $N$ was studied, improving an earlier result of Raikov.

Furthermore, a related problem of Erdős is also examined: How dense can a set of integers be, if none of them divides the product of $h$ others? Let $F_{h}(n)$ denote the maximal possible size of such a subset of $\{1,2, \ldots, n\}$. The case $h=2$, that is, such sets of integers where none of the elements divides the product of two others, was settled by Erdős [172]. Chan, Györi and Sárközy [168] studied the case $h=3$. Furthermore, recently Chan [167] determined the order of magnitude of $F_{h}(\mathrm{n})-\pi(n)$ for every fixed $h$. Pach and Sándor strengthened this result by giving such lower and upper bounds for $F_{h}(n)-\pi(n)$ that differ only by a constant factor not depending either on $h$ or on $n$ :

$\pi(n)+0.2 n^{2 /(h+1)} / \log ^{2}(n)<F_{h}(\mathrm{n})-\pi(n)<\pi(n)+443 n^{2 /(h+1)} / \log ^{2}(n)$.

They also studied the infinite version of Erdős's question, for instance, proved that any subset $A$ of positive integers satisfying the condition must be thin sometimes: It cannot contain at least $\pi(n)+n^{\varepsilon}$ many elements of $\{1,2, \ldots, n\}$ for every $n$.

\subsection{Summary}

Current research in two areas have been presented in order to give an impression on the multifaceted research activities performed at our department. We were glad to learn that Dr. Péter Pál Pach, the author of the results in Section 3 has recently been awarded the prestigious Géza Grünwald Prize of the János Bolyai Mathematical Society in December 2015 and won the János Bolyi Research Scholarship of the Hungarian Academy of Sciences.

Most of the research activities reported in this article are supported by the OTKA grant 108947.

\section{Department of Telecommunications and Media Informatics}

The Department of Telecommunication and Media Informatics has been hosting activities in data science, content technologies, Internet and cloud communications, engineering management, cognitive info-communications, Internet of Things, speech technology and smart interactions, not only in engineering but also in a multidisciplinary manner. In this 2016 annual research report, we commend these valuable activities to the readers' honorable attention.

\subsection{Data Science and Content Technologies}

In data science, our most recent research is about the renewable energy forecast by using distributed and privacy preserved machine-learning methods $[180,181]$. At end of the year, a successful R\&D project was finished with OTP Bank, in which a new interpretable classification method was created and implemented to increase the efficiency of prediction. Furthermore, our collages obtained new competencies in projects by analyzing GPS tracking dataset, mining of parcel logistic databases, processing financial transaction logs to detect fraud and analyzing process control data of an oil refinery. Most of them contained task to build Spark based big data infrastructure as well.

We elaborated a complex system [179] to detect, classify and track objects (fishes) in underwater video by examining each image frame of it. We used Kalman filter to track the moving objects, and Hungarian method was used to match the pair of the objects in consecutive time periods because of many fishes. We categorized the detected fishes with C-SVC classifier, as an advanced SVM (Support Vector Machine) classifier. As further improvement, we used color histograms and discriminant training method for filtering out false detections. For whale individual recognition we elaborated another system to compare the individuals by applying BoW (Bag of Word) model, using Harris-Laplace detector and dense SIFT for creating low-level features; after that GMM (Gaussian Mixture Model) based Fisher vectors were calculated and compared to each other for the recognition. In addition to this, we applied background segmentation as pre-processing [179]. Another research area was active learning in multimedia mining, which solves the manual labeling problem by reducing the number of images that are need to labeled. It selects a subset of the whole data by iteratively querying the unlabeled instances based on their predicted information. One way to estimate the information content of an image is using uncertainty sampling as query strategy, and we improved this method by balancing the distribution of the already labeled images. In the multimedia research area, there was another work: together with Speech Technology and Smart Interactions Laboratory group, we worked out solutions (SVM and convolutional neural network) for classification of plant images to given species [202]. 


\subsection{Internet, Cloud Communications, loT Analytics and Software Defined Networking}

The High Speed Networks Laboratory (HSNLab) as a strategic partner of Ericsson has been performing the following closely Ericsson related research projects: SmartActive SquashIoT Sport Analytics, Deterministic Networks-Time Sensitive Networking, Software based Ternary Content Addressable Memories, Optimal Cloud, Dataplane. Furthermore, the lab takes part in the following large EU projects: COMBO, UNIFY, 5GEx. In connection with and also beyond these projects, the main research achievements are as follows.

For Smart Spaces (IoT) environments we designed and analyzed adaptive event-driven sampling strategies which can efficiently treat data collection, processing and transfer with physical constraints $[183,184]$. We contributed to establishing and analyzing an end-to-end multi-domain management and orchestration frameworks for software defined infrastructures [182, 185]. We further elaborated our research in digital fountain based coding congestion handling. We created a novel transport mechanism called Digital Fountain Based Communication Protocol and performed a detailed performance evaluation of it [186]. In [187] we have had a curious contribution of establishing a general framework for kd trees and quad trees, which could be a useful construction in theoretical computer science. In 2016, we also took part in a great editorial created a special issue of the prestigious journal of Photonic Network Communications [188]. In [189] we presented our vision of creating a 5G Exchange for enabling the orchestration of networking and service resources in an automated way across technological and administrative domains. We identified the necessary next steps in terms of implementation and experimentation, and proposed a sandbox environment where partners may experiment with life-size multi-domain network orchestration.

In the MTA-BME Future Internet research group (part of HSNLab) we gained the following research results. Packet classification is an important building block in many network services. We investigated the applicability of lossy compression to create packet classifiers requiring much less memory than the theoretical size limits of the semantically-equivalent representations, enabling significant reduction in their cost and power consumption [190]. In survivable routing diversity, coding provides a nice trade-off between the simplicity of dedicated protection and bandwidth-efficiency of network coding to ensure instantaneous recovery for the connections. We thoroughly investigated the optimal structure of diversity coding-based survivable routing, identified the delays of the underlying acyclic graphs and perform complexity analysis, exact and heuristic solutions for these delay aware survivable routing problems [191]. Together with Ericsson experts, we introduced ESWITCH, a novel switch architecture that uses on-the-fly template-based code generation to compile any OpenFlow pipeline into efficient machine code. We presented a proof-of-concept prototype and we demonstrated on illustrative use cases that ESWITCH yields a simpler architecture, superior packet processing speed, improved latency and CPU scalability, and predictable performance. Our prototype can easily scale beyond 100 Gbps on a single Intel blade even with complex OpenFlow pipelines [192]. We have also taken part in organizing several international conferences, e.g. IEEE ONDM 2016 [188], IEEE EUSIPCO 2016; we were TPC members in IEEE Infocom 2016.

\subsection{Internet of Things Platforms and Applications}

This year a new laboratory established in our department with the name Smart Communications Laboratory (SmartCom Lab) with leading activities in the following projects.

This year we retain our central role in Europe's largest automation project, Arrowhead, which extensively relies on our expertise in IP-based communication and in architecting collaborating platforms. We have substantial contribution to setting up the requirements for enabling interoperability and integrability of services provided by IoT devices. The overall concept for interoperability within Arrowhead is described in [15], providing the motivation behind IoT in industrial automation. The benefit of choosing Arrowhead instead of other frameworks is that it supports real-time applications, QoS-bound applications, and applications with various security requirements by design. Furthermore, Arrowhead is already deployed in dozens of products, and proved its capabilities in another few dozen industrial demonstrations. We keep improving the framework in many aspects: integrated security by ticketing and certificate-based approaches [194], deployment support through advanced plant descriptions, inter-cloud service orchestration [195], and complex use-case demonstrations, including the multi-stakeholder electro-mobility domain.

Together with several industrial and academic partners we have also worked out solutions for cloud based automation technologies and applications. We are also active participants in MANTIS, a large EU project for developing a Cyber Physical System (CPS) based proactive maintenance service platform architecture. This year we contributed to establish IoT platforms for data collection and processing [196], which is an important prerequisite of an optimized CPS based maintenance system. These systems form part of a larger network of heterogeneous and collaborative systems (e.g. vehicle fleets or photovoltaic and windmill parks) connected via robust communication mechanisms able to operate in challenging environments.

The sponsored research agreement between SmartCom Lab and IBM Research Zürich has started this year, based on our results provided by our FPGA-based monitoring equipment [197, 198]. The target of the project is data center monitoring, with two main tracks: (i) intrusion detection and protection systems (ISD/IPS), and (ii) support for Software Defined Networking (SDN). The core idea of the concept is that the monitoring is supported by smart mirroring capabilities of modern switch/ 
routers, and the mirrored traffic is processed by hardware accelerated monitoring units. This setup allows sub-millisecond reaction time for IDS/IPS. The concept has been verified through practical live demos on live traffic from a big Hungarian network infrastructure provider. The SDN track of the research aims at supporting digested, structured monitoring data about network status, even with a sub-millisecond granularity.

Furthermore, SmartCom Lab is working the definition, design and development of a quality measurement system for Internet users within Hungary. The system is responsible for carrying out on-request measurements of network and service performance, initiated from the subscriber endpoints. Measured metrics include upload and download speed, latency, packet delay variation, packet loss ratio, and other QoS metrics.

\subsection{Speech Communication, Smart Interactions and Deep Learning}

The Speech Technology and Smart Interactions Lab (SmartLab) has been actively participating in an international research co-operation project led by Idiap Research Institute. As a result, we proposed an innovative speech vocoder with continuous parameters [199] and applied this both in Hidden Markov-model (HMM) based and Deep Neural Network based [200] speech synthesis. HMM-based speech synthesis has been extended with the modeling of short and interrogative sentences [201]. We intend to increase the quality of text-to-speech synthesis by introducing stress-labels extracted from speech waveform in statistical parametric speech synthesis [207]. SmartLab won both NVIDIA GPU Hardware and NVIDIA GPU Education Center Grants. We used the acquired GPUs for our recent deep learning research and education. Concerning research speech synthesis [200], sound and image [202] processing papers were published, as well as an overview of deep learning based speech synthesis [203]. In education, both MSc and BSc students work on deep learning related topics. A deep learning class -'Deep Learning in practice with Python and LUA"- was also initiated with more than 40 students. The largest scale application of the Lab is the polyglot domain-optimized textto-speech system for the Hungarian Railways that has been in operation at more than 60 railway stations and stops.

The Speech Recognition and Audio Mining Laboratory (ASRLab) puts its focus on broadcast news and spontaneous speech recognition of CEE languages as well. Moreover, in cooperation with SmartLab it has broadened its portfolio with audio fingerprint detection for monitoring television and radio broadcasts. Our basic research aim is robust, very large vocabulary continuous speech recognition in real time - in other words generalized speech-to-text. The sub-fields supporting this aim are as follows: Acoustic modeling: deep neural networks for modeling speech sounds and pronunciations, semi-supervised techniques for cost effective acoustic model training, language independent technologies (grapheme-based acoustic modeling). Language modeling: morph based and very large vocabulary word based methods for morphologically rich languages (e.g. Uralic, Turk or Slavic languages). Generative and discriminative language models. Feature extraction and signal processing: nonlinear and deep learning methods for acoustic feature extraction. Speech-music separation, speech endpoint detection, channel compensations, audio fingerprint generations. Possible applications include call-center speech transcription and analytics, voice routing, real-time closed captions for TV broadcasts, content based indexing of large multimedia archives and dictation systems. The application of LVCSR (Large Vocabulary Continuous Speech Recognition) technology was investigated for real-time, resource-limited broadcast close captioning. The work focused on transcribing live broadcast conversation speech to make such programs accessible to deaf viewers. This solution is already integrated into the TV broadcast and Teletext system of the Hungarian Media Support and Asset Management Fund (MTVA).

The Laboratory of Speech Acoustics (LSA) has performed research on speech signal processing, using speech as bio signal. The aim of this research to construct different automated medical decision support systems, which are based on speech processing [205]. For example, a depression detection system will be one of the potential biomarkers for the severity of depression will also serve as an indicator for the response to treatment. A related topic is the examination of the sensitivity of acoustic-phonetic parameters of speech to hypoxia and to Seasonal Affective Disorder and the definition of a metric that alert crews at early stage of cognitive dysfunction (Automatic detection) using Concordia Antarctic Station as Human Exploration Analogue. This research is financed by the European Space Agency, ESA [206]. Now the central theme of the research, how it is possible separate the speech parameters into language-specific and language-independent groups that is how the three above-mentioned issues can be solved in a language-independent way. The other main research direction is a Computer-Assisted Prosody Pronunciation Teaching System, what will teach supra-segmental parameters as intonation, stress and speech rhythm. Prosody modelling is one of the most up to date topics in the speech community, and our prosody modelling research is performed in strong cooperation between the SmartLab and LSA [207].

\subsection{Engineering Management and Cognitive Info-communications}

The Engineering Management Laboratory (EMLab) plays central role in performing the $3^{\text {rd }}$ Hungarian Future Internet Conference (MJIK 2016) focused on the next generation Internet technologies and the Smart City issues. The MJIK 2016 was organized by the EMLab and held at November 10, in Budapest. The programme of MJIK 2016 comprised presentations from foreign and domestic speakers involving scientists from our department. A special issue of Híradástechnika including 
selected papers of MJIK2015 was edited, too. A smart internet ecosystem model was also elaborated; the related Digital Single Market and regulatory issues were studied.

The Cognitive Mediainformatics Laboratory, besides several conference and journal publications presented this year a book on TP model transformation, which turned out to be a useful fundamental tool for creating designing framework in control systems [208].

\subsection{Summary}

The scientific and research activities of TMIT in 2016, since many years as well, have been substantial, valuable and highly influential in both domestic and international professional circles. Inevitably, this review cannot be intended to be exhaustive, for more details and deeper insight, we ask the kind readers to visit our home page: www.tmit.bme.hu.

\section{Department of Electric Power Engineering}

There are several projects at the department, which are the continuation of the research activity from the previous year, while others are new ones started in 2017.

\subsection{Beyond State-of-the-art Technologies for Re-powering AC Corridors and Multi-terminal HVDC Systems (BEST PATHS)}

"Best Paths" project is one of the latest, but highest EU FP 7 projects. It consist of different parts (http://www.bestpaths-project.eu).

In the High Voltage Laboratory of Budapest University of Technology and Economics, two main topics are concerned within the confines of the BEST PATHS project, which aims to develop novel network technologies to increase the pan-European transmission network capacity and electricity system flexibility.

One of them is the live-line maintenance related to the high voltage transmission overhead line, which is also a main research area of the Laboratory for several decades. New type of equipment such as the design of mounting chair and a conductor car from an innovative approach is included. With the use of the new main equipment and additional new tools, the available live working technologies are expanded to the new type of compact $400 \mathrm{kV}$ towers including the changing of insulator strings.

Another main topic is the research and development of dynamic line rating of high voltage overhead lines. This technique can exploit the additionally available capacity of high voltage overhead lines, which cause more efficient use of the prepared network section. A further significant advantage of this method is that it helps to overcome the extra load due to icing. The project started with research and the aim of it is to build a usable system for this task.

\subsection{Examination of Dielectric Properties of New Type Oils}

The main aim of this project is to investigate the usability of new type of oils nevertheless the determination electrical properties of different kind of oils - including environmentally friendly - used for a part of insulation systems. The work includes several thousands of measurements of spark over voltage and ignition voltage of partial discharges in case of different length of samples, the statistical processing of the values, and the validation of the measured values, which requires special calculation methods and the determination of the distribution of electric field in each case functionally with the application of finite element method.

\subsection{Investigation of Gas Insulated High Voltage Devices}

At the late twentieth century, the use of special gases became more common instead of the use of oil, high-pressure air and vacuum in high voltage circuit breakers, such as SF6. With the use of SF6, the decreasing of sizes of the switching and measuring devices became possible, because of its outstanding dielectric properties; therefore, the use of gas-insulated substations (GIS) has increased over. In order to research of further opportunities of the possible development of this equipment the behavior of dielectric properties was examined of GIS with the application of special coating on the surface of electrodes with different surface roughness was in the high voltage laboratory. The results of the research and measurement were used for the design of a new type of gas-insulated device.

\subsection{Medium Voltage Equipment on the Overhead Lines}

The $20 \mathrm{kV}$ distribution system of the Hungary contains of several ten thousands kilometer overhead line, which means several piece of network elements such as pylons, insulators, surge arresters, connectors, sleeves, etc. To build and hold a sustainable grid it is essential to fit the most reliable elements. For the selection of the most reliable equipment preliminary examinations should be performed - which can include accelerated aging, destructive and non-destructive examinations with specific regard to the new types of equipment in which cases there are no available long-term experiences. One of the main purposes to compare the well-known standardized methods and techniques to testing each equipment and find the most reliable way to diagnose the weaknesses.

\subsection{Development of an Expert System for Determination of Health Index}

Investigation of diagnostic method of primary equipment (such as transformers, circuit breakers, current transformers in HV/MV substations) and the development a diagnostic strategy, guideline and the development an expert system for 
primary equipment are the main purposes of this project. The project work has several steps:

- Based on investigated method establishing a priorization method which will be the basis of comparison for different primary equipment (in terms of type, location, and environment).

- Based on method development of expert system, which will be applicable for decision making concerning substation investment proposals.

- Based on the theoretical background the development of an expert system software.

As a result of the carried out work, with the help of health index and the built expert system a more efficient lifetime management can be realized for primary equipment.

\subsection{Development of Education of Live Line Maintenance}

Nowadays the different methods of Live-Line Maintenance (LLM) are widely used during the operation of transmission and distribution network due to its short and long-term advantages. The statistics shows that during LLM less accidents happen than in case of another techniques including de-energized work. A significant affecting factor of that is the high degree of regulation of live line work, and the qualified workers, therefore the training is a very important step. The High Voltage Laboratory trains different working methods on medium and high voltage systems co-operated with the Hungarian TSO and DSOs also.

\section{References}

[1] Szabó, M., Pomázi, K. D., Radostyán, B., Szegletes, L., Forstner, B. "Estimating Task Difficulty in Educational Games." In: 7th IEEE Conference on Cognitive Infocommunications, Wroclaw, Poland, Oct. 16-18, 2016. pp. 397-402. https://doi.org/10.1109/CogInfoCom.2016.7804582

[2] Radostyán, B., Forstner, B., Szegletes, L., Pomázi, K. D., Gazdi, L. "Adaptive Figural Abstraction Test with Generated Exercises." In: 7th IEEE Conference on Cognitive Infocommunications, Wrocław, Poland, Oct. 16-18, 2016. pp. 327-330. 2016. https://doi.org/10.1109/CogInfoCom.2016.7804570

[3] Dunaev, D., Lengyel, L. “An Intermediate Level Obfuscation Method.” Acta Polytechnica Hungarica. 11 (7), pp. 179-195. 2014. https://doi.org/10.12700/APH.11.07.2014.07.11

[4] Lengyel. L., Ekler, P., Tömösvári, I., Balogh, T., Mezei, G., Forstner, B., Charaf, H. "Model-driven Multi-Domain IoT." In: Emerging Trends and Applications of the Internet of Things. Chapter 6. (P. Kocovic, P., Ramachandran, M., Behringer, R., Mihajlovic, R. (Eds)). pp. 167-190. IGI Global, 2017. https://doi.org/10.4018/978-1-5225-2437-3.ch006

[5] Lengyel, L., Ekler, P., Ujj, T., Balogh, T., Charaf, H. "SensorHUB - An IoT Driver Framework for Supporting Sensor Networks and Data Analysis." International Journal of Distributed Sensor Networks. Vol. 2015, Article ID 454379, 12 p. 2015.

[6] EIT Climate-KIC. "Knowledge \& Innovation Community." 2016. URL: http://www.climate-kic.org.

[7] Anon. "Sustainable Outdoor Lighting \& Sensory Urban Networks" 2016. URL: http://www.solsun.co.uk/index.php/SOLSUN
[8] Csorba, K., Barancsuk, L., Blázovics, L. "Visual Traffic Load Sensor for Emission Estimation.” PROCEDIA ENGINEERING. 168, pp. 47-50. 2016. https://doi.org/10.1016/j.proeng.2016.11.139

[9] BME AUT. "cv4sensorhub. Image processing for interdisciplinary applications.” 2016. URL: http://bmeaut.github.io/cv4sensorhub/

[10] Csorba, K., Barancsuk, L., Székely, B., Zöldföldi, J. “GrainAutLine - A Supervised Grain Boundary Extraction Tool Supported by Image Processing and Pattern Recognition." In: Proceedings of Association for the Study of Marble \& Other Stones in Antiquity, XI. International Conference, Split, Croatia. pp. 95-96. 2015.

[11] Pandi, S., Fitzek, F. H. P., Lehmann, Ch., Nophut, D., Kiss, D., Kovács, V., Nagy, Á., Csorvási, G., Tóth, M., Rajacsis, T., Charaf, H., Liebhart, R. "Joint Design of Communication and Control for Connected Cars in 5G Communication Systems." In: Proceedings of IEEE Globecom Workshops, Washington, USA. pp. 1-7. 2016 https://doi.org/10.1109/GLOCOMW.2016.7848940

[12] Braun, P. J., Ekler, P., Fitzek, F. H. P. "Demonstration of a P2P assisted video streaming with WebRTC and Network Coding." In: $14^{\text {th }}$ IEEE Annual Consumer Communications \& Networking Conference (CCNC), Las Vegas, U.S.A., Jan, 2017.

[13] Braun, P. J., Ekler, P., Fitzek, F. H. P. “Network coding enhanced browser based Peer-to-Peer streaming.” In: 2016 IEEE International Conference on Systems, Man, and Cybernetics, Budapest, Hungary, 2016. https://doi.org/10.1109/SMC.2016.7844550

[14] Braun, P. J., Pand, S., Schmoll, R. S., Fitzek, F. H. P. "On the Study and eployment of Mobile Edge Cloud for Tactile Internet using a 5G Gaming application." In: $14^{\text {th }}$ IEEE Annual Consumer Communications \& Networking Conference (CCNC), Las Vegas, USA, 2017.

[15] Pand, S., Schmoll, R. S., Braun, P. J., Fitzek, F. H. P. "Demonstration of Mobile Edge Cloud for Tactile Internet using a 5G Gaming Application.” In: $14^{\text {th }}$ IEEE Annual Consumer Communications \& Networking Conference (CCNC), Las Vegas, USA, 2017.

[16] Sipos, M., Gahm, J., Venkat, N., Oran, D. "Erasure coded storage on a changing network: the untold story.” In: 2016 IEEE Global Communications Conference (GLOBECOM), Dec, 2016. pp. 1-6. https://doi.org/10.1109/GLOCOM.2016.7841820

[17] Tomoskozi, M., Fitzek, F. H. P., Lucani, D. E., Pedersen, M. V., Seeling, P. "On the delay characteristics for point-to-point links using random linear network coding with on-the-fly coding capabilities.” In: European Wireless 2014; 20th European Wireless Conference; Proceedings of, Barcelona, Spain, May 14-16, 2014. pp 1-6.

[18] Tomoskozi, M., Fitzek, F. H. P., Lucani, D. E., Pedersen, M. V., Seeling, P., Ekler. P. "On the packet delay characteristics for serially-connected links using random linear network coding with and without recoding." In: European Wireless 2015; 21th European Wireless Conference; Proceedings of, Budapest, Hungary, May 20-22, 2015. pp 1-6.

[19] Sipos, M., Braun, P. J., Lucani, D. E., Fitzek, F. H. P., Charaf, H. “On the Effectiveness of Recoding-based Repair in Network Coded Distributed Storage." Periodica Polytechnica-Electrical Engineering and Computer Science. 61 (1), pp. 12-21. 2017. https://doi.org/10.3311/PPee.9377

[20] Tomoskozi, M., Seeling, P., Ekler, P., Fitzek, F. H. P. "Performance prediction of robust header compression version 2 for RTP audio streaming using linear regression.” In: European Wireless 2016 (EW2016), Oulu, Finland, May, 2016.

[21] Tomoskozi, M., Seeling, P., Ekler, P., Fitzek, F. H. P. "Efficiency gain for RoHC compressor implementations with dynamic configuration." In: VTC2016-Fall Workshop on Cellular Internet of Things - Emerging Trends and Enabling Technologies, Sept, 2016. 
[22] Tomoskozi, M., Seeling, P., Ekler, P., Fitzek, F. H. P. "Performance evaluation of network header compression schemes for UDP, RTP and TCP." Periodica Polytechnica-Electrical Engineering and Computer Science. 60(3), pp. 151-162. 2016. https://doi.org/10.3311/PPee.8958

[23] Tomoskozi, M., Ekler, P. "Analysis of robust header compression version 2 compressible packets for utility prediction with Bayesian regression." In: Automation and Applied Computer Science Workshop, 2016.

[24] Tomoskozi, M., Seeling, P., Ekler, P., Fitzek, F. H. P. "Regression model building and efficiency prediction of RoHCv2 compressor implementations for VoIP." In: 2016 IEEE Global Communications Conference: Mobile and Wireless Networks (Globecom2016 MWN), Washington, USA, 2016. https://doi.org/10.1109/GLOCOM.2016.7842207

[25] Járdán, R. K., Stumpf, P., Varga, Z., Endisch, Ch., Sipos, P., Simon, M. "Laboratory system for measurement of iron losses in high speed drives." International Journal of Hydrogen Energy. 41(29), pp. 1265012658. 2016. https://doi.org/10.1016/j.ijhydene.2016.01.061

[26] Rakos, B. "Pulse-Driven, Photon-Coupled, Protein-Based Logic Circuits." Advances in Intelligent Systems and Computing.. 519, pp. 123127, 2016. https://doi.org/10.1007/978-3-319-46490-9_18

[27] Jani, L., Poppe, A. "Improved Method for Logi-Thermal Simulation With Temperature Dependent Signal Delay.” In: Proceedings of the $22^{\text {nd }}$ International Workshop on THERMal INvestigation of ICs and Systems (THERMINIC'16), Budapest, Hungary, Sep. 21-23, 2016. (Poppe, A. (Ed.)), pp. 302-306. https://doi.org/10.1109/THERMINIC.2016.7749071

[28] Szalai, A., Szabó, T., Horváth, P., Tímár, A., Poppe, A. "SmartSSL: Application of IoT/CPS design platforms in LED-based street-lighting luminaires." In: Proceedings of the VI. IEEE Lighting Conference of the Visegrad Countries LUMEN V4, Sep. 13-16, 2016, Karpacz, Poland. pp. 65-70. https://doi.org/10.1109/LUMENV.2016.7745518

[29] Hegedüs, J., Poppe, A. "Simulation of luminaires based on chip level multi-domain modeling of power LEDs." In: Proceedings of the VI. IEEE Lighting Conference of the Visegrad Countries LUMEN V4, Sep. 13-16, 2016, Karpacz, Poland. pp. 59-64. https://doi.org/10.1109/LUMENV.2016.7745517

[30] Hegedüs, J., Hantos, G., Poppe, A. "Embedded Multi-domain LED Model for Adaptive Dimming of Streetlighting Luminaires.” In: Proceedings of the 22nd International Workshop on THERMal INvestigation of ICs and Systems (THERMINIC'16), Budapest, Hungary, Sep. 21-23, 2016. pp. 208-212. https://doi.org/10.1109/THERMINIC.2016.7749053

[31] Takács, G., Bognár, Gy., Bándy, E., Rózsás, G., Szabó, P. G. "Fabrication and Characterization of Microscale Heat Sinks." In: Proceedings of the $22^{\text {nd }}$ International Workshop on THERMal INvestigation of ICs and Systems (THERMINIC'16), Budapest, Hungary, Sep. 21-23, 2016. (Poppe, A. (Ed.)), pp. 264-267. https://doi.org/10.1109/THERMINIC.2016.7749062

[32] Bognár, Gy., Takács, G., Pohl, L., Szabó, P. G. "Thermal modelling of integrated heatsink structures." In: Proceedings of the Symposium on Design, Test, Integration and Packaging of MEMS/MOEMS (DTIP'16), Budapest, Hungary, 31 May-2 June 2016. (Charlot, B., et al. (Eds)). pp. 173-177. https://doi.org/10.1109/DTIP.2016.7514863

[33] Hantos, G., Hegedüs, J., Rencz, M., Poppe, A. “Aging Tendencies of Power MOSFETs - A Reliability Testing Method Combined with Thermal Performance Monitoring." In: Proceedings of the $22^{\text {nd }}$ International Workshop on THERMal INvestigation of ICs and Systems (THERMINIC'16), Budapest, Hungary, Sep. 21-23, 2016. (Poppe, A. (Ed.)). pp. 220-223. https://doi.org/10.1109/THERMINIC.2016.7749055

[34] Cardoso, A. G., Barros, H., Hantos, G. "Fabrication, Performance and Reliability of a Thermally Enhanced Wafer Level Fan Out Demonstrator with Integrated Heatsink." In: Proceedings of the $22^{\text {nd }}$ International Workshop on THERMal INvestigation of ICs and Systems (THERMINIC'16), Budapest, Hungary, Sep. 21-23, 2016. (Poppe, A. (Ed.)). pp. 336-344. https://doi.org/10.1109/THERMINIC.2016.7749079
[35] Poppe, A., Hegedüs, J., Szalai, A. "Multi-domain modeling of power LEDs based on measured isothermal I-V-L characteristics." In: Proceedings of the CIE Lighting Quality \& Energy Efficiency Conference, Melbourne, Australia, Mar. 3-5, 2016. pp. 318-327.

[36] Poppe, A., Hegedüs, J., Szalai, A., Bornoff, R., Dyson, J. “Creating multiport thermal network models of LED luminaires for application in system level multi-domain simulation using Spice-like solvers." In: Proceedings of the 32nd IEEE Semiconductor Thermal Measurement and Management Symposium (SEMI-THERM'16), San Jose, USA, Mar. 14-17, 2016. pp. 44-49. https://doi.org/10.1109/SEMI-THERM.2016.7458444

[37] Poppe, A. "From Measurements to Standardized Multi-Domain Compact Models of LEDs: Towards predictive and efficient modeling and simulation of LEDs at all integration levels along the SSL supply chain." In: Proceedings of the $15^{\text {th }}$ International Symposium on the Science and Technology of Lighting (LS15), Kyoto, Japan, May 22-27, 2016. pp. 387-392.

[38] Bornoff, R., Hildenbrand, V., Lugten, S., Martin, G., Marty, Ch., Poppe, A., Rencz, M., Schilders, W., Yu, J. "Delphi4LED - From Measurements to Standardized Multi-Domain Compact Models of LEDs: a New European R\&D Project for Predictive and Efficient Multi-domain Modeling and Simulation of LEDs at all Integration Levels Along the SSL Supply Chain." In: Proceedings of the $22^{\text {nd }}$ International Workshop on THERMal INvestigation of ICs and Systems (THERMINIC'16), Budapest, Hungary, Sep. 21-23, 2016. (Poppe, A. (Ed.)). pp. 174-180. https://doi.org/10.1109/THERMINIC.2016.7749048

[39] Mizsei, J., Lappalainen, J., Ur, S., Németh, M., Gaál, L. "Thermal-Electronic Device and Integrated Circuit.” In: Proceedings of the 16th IEEE International Conference of Nanotechnology, Sendai, Japan, Aug. 22-25, 2016. pp. 966-969. https://doi.org/10.1109/NANO.2016.7751427

[40] Ur, S., Mizsei, J., Pohl, L. "Modelling of the Thermoelectrical Performance of Devices Based on VO2." In: Proceedings of the $22^{\text {nd }}$ International Workshop on THERMal INvestigation of ICs and Systems (THERMINIC'16), Budapest, Hungary, Sep. 21-23, 2016. (Poppe, A. (Ed.)). pp. 307-310. https://doi.org/10.1109/THERMINIC.2016.7749072

[41] Horváth, P., Hosszú, G., Kovács, F. “Semi-Automatic RTL Methods for System-on-Chip IP Delivery in the Cyber-Physical System Era." Periodica Polytechnica Electrical Engineering and Computer Sciences. 60(2), pp. 107-124. 2016. https://doi.org/10.3311/PPee.8527

[42] Jani, L., Poppe, A. "Multilevel logic and thermal co-simulation." Microelectronics Reliability. 67, pp. 46-53. 2016. https://doi.org/10.1016/j.microrel.2016.08.019

[43] Takács, G., Szabó, P. G., Bognár, Gy. "Enhanced thermal characterization method of microscale heatsink structures." Microelectronics Reliability. 67, pp. 21-28. 2016. https://doi.org/10.1016/j.microrel.2016.09.019

[44] Takács, G., Szabó, P. G., Bognár, Gy. "Modelling of the flow-rate dependent partial thermal resistance of integrated microscale cooling structures." Microsystem Technologies. pp. 1-10. 2016. https://doi.org/10.1007/s00542-016-2879-2

[45] Martin, G., Poppe, A., Lungten, S., Heikkinen, V., Yu, J., Rencz, M., Bornoff, R. "Delphi4LED - From Measurements to Standardized Multi-Domain Compact Models of Light Emitting Diodes (LED)." ELECTRONICS COOLING. 22 (12), pp. 20-23. (2016)

[46] Lavrenko, T., Marzinzig, K., Walter, T., Plesz, B., Ress, S. "On the application of the vibrating Kelvin probe method for quality control of $\mathrm{Cu}(\mathrm{In}, \mathrm{Ga})(\mathrm{Se}, \mathrm{S}) 2$ thin-film solar modules." Progress in Photovoltaics: Research and Application. 24(12), pp. 1554-1565. 2016. https://doi.org/10.1002/pip.2746

[47] Mizsei, J., Lappalainen, J. "Thermal-electronic integrated circuits using thermally sensitive VO2 MIT material." Procedia Engineering. 168, pp. 1070-1073. 2016. https://doi.org/10.1016/j.proeng.2016.11.342 
[48] Ender, F., Weiser, D., Poppe, L. "Microfluidic multiple cell chip reactor filled with enzyme-coated magnetic nanoparticles." In: Lab-on-a-Chip Fabrication and Application. (Stoytcheva, M., Zlatev, R. (Eds.)), InTech Open Publisher. 2016. https://doi.org/10.5772/62512

[49] Horváth, P. "System level synthesis method for automated RTL design." (Rendszerszintű szintézis módszer automatizált RTL tervezéshez.) PhD dissertation (electrical engineering), Budapest University of Technology and Economics, Faculty of Electrical Engineering and Informatics, 2016. (in Hungarian)

[50] Ender, F. "Modeling, Realization and Characterization of Microreactors in Lab-on-a-Chip Devices." PhD dissertation (electrical engineering), Budapest University of Technology and Economics, Faculty of Electrical Engineering and Informatics, 2016.

[51] Plesz, B. "Investigation of the temperature dependence of thin photoactive layer crystalline silicon solar cells." $\mathrm{PhD}$ dissertation (electrical engineering), Budapest University of Technology and Economics, Faculty of Electrical Engineering and Informatics, 2016. (in Hungarian)

[52] Hutli, E., Bonyár, A., Oszetzky, D., Nedeljkovic, M. S. "Plastic Deformation and Modification of Surface Characteristics in Nano and Micro Levels and Enhancement of Electric Field of FCC Materials Using Cavitaion Phenomenon." Mechanics of Materials. 92, pp. 289-298. 2016. https://doi.org/10.1016/j.mechmat.2015.10.006

[53] Hutli, E., Nedeljkovic, M. S., Bonyár, A., Radovic, N. A., Llic, V., Debeljkovic, A. "The Ability of Using the Cavitation Phenomenon as a Tool to Modify the Surface Characteristics in Micro and in Nano Level." Tribology International. 101, pp. 88-97. 2016. https://doi.org/10.1016/j.triboint.2016.04.006

[54] Hutli, E., Nedeljkovic, M. S., Bonyár, A., Légrády, D. "Experimental Study on the Influence of Geometrical Parameters on the Cavitation Erosion Characteristics of High Speed Submerged Jets." Experimental Thermal and Fluid Science. 80, pp. 281-292. 2017.

https://doi.org/10.1016/j.expthermflusci.2016.08.026

[55] Hutli, E., Nedeljkovic, M. S., Radovic, N. A., Bonyár, A. "The Relation between the High Speed Submerged Cavitating Jet Behavior and the Cavitation Erosion Process." International Journal of Multiphase Flow. 83, pp. 27-38. 2016. https://doi.org/10.1016/j.ijmultiphaseflow.2016.03.005

[56] Bonyár, A. "AFM characterization of the shape of surface structures with localization factor." Micron. 87, pp. 1-9. 2016. https://doi.org/10.1016/j.micron.2016.05.002

[57] Hurtony, T., Bonyár, A., Gordon, P., Harsányi, G. „Investigation of intermetallic compounds (IMCs) in electrochemically stripped solder joints with SEM.” Microelectronics Reliability. 52 (6), pp. 1138-1142. 2012. https://doi.org/10.1016/j.microrel.2011.12.035

[58] Hurtony, T., Szakál, A., Almásy, L., Len, A., Kugler, S., Bonyár, A., Gordon, P. „Characterization of the microstructure of tin-silver lead free solder." Journal of Alloys And Compounds. 672, pp. 13-19. 2016. https://doi.org/10.1016/j.jallcom.2016.02.177

[59] Garami, T., Krammer, O. "Quantitative analyses of Ag3Sn intermetallic compound formation in $\mathrm{SnAgCu}$ solder alloys." Journal of Materials Science: Materials in Electronics. 26(11), pp. 8540-8547. 2015. https://doi.org/10.1007/s10854-015-3526-x

[60] Garami, T., Krammer, O., Harsányi, G., Martinek, P. "Method for validating CT length measurement of cracks inside solder joints." Soldering \& Surface Mount Technology. 28 (1), pp. 13-17. 2016. https://doi.org/10.1108/SSMT-10-2015-0029

[61] Tamási, P., Kósa, Gy., Szabó, B., Berényi, R., Medgyes, B. "Effect of Bismuth and Silver on the Corrosion Behavior of Lead-free Solders in $3.5 \mathrm{wt} \% \mathrm{NaCl}$ Solution." Periodica Polytechnica-Electrical Engineering and Computer Science. 60(4), pp. 232-236. 2016.

https://doi.org/10.3311/PPee.9706
[62] Skwarek, A., Illés, B., Géczy, A. "Characterization of Tin Pest by Electrical Resistance Measurement." In: Proceedings of IEEE 22nd International Symposium for Design and Technology in Electronic Packaging (SIITME). pp. 314-319. 2016.

https://doi.org/10.1109/SIITME.2016.7777298

[63] Illés, B., Géczy, A. "Investigating the heat transfer on the top side of inclined printed circuit boards during vapour phase soldering." Applied Thermal Engineering. 103, pp. 1398-1407. 2016.

https://doi.org/10.1016/j.applthermaleng.2016.04.153

[64] Illés, B., Géczy, A., Skwarek, A., Busek, D. "Effects of substrate thermal properties on the heat transfer coefficient of vapour phase soldering." International Journal of Heat And Mass Transfer. 101, pp. 69-75. 2016. https://doi.org/10.1016/j.ijheatmasstransfer.2016.04.116

[65] Bušek, A., Fazekas, L., Géczy, A., Plaček, M. "Substrate influence on temperature distribution along the PCB during vapour phase soldering." In: Proceedings of IEEE 39th International Spring Seminar on Electronics Technology, pp. 267-272. 2016.

https://doi.org/10.1109/ISSE.2016.7563203

[66] Géczy, A., Kvanduk, B, Illés, B., Harsányi, G. "Comparative Study on Proper Thermocouple Attachment for Vapour Phase Soldering Profiling." Soldering \& Surface Mount Technology. 28(1), pp. 7-12. 2016. https://doi.org/10.1108/SSMT-10-2015-0033

[67] Géczy, A., Gál, L., Kovács, B., Hajdu, I. “Toward producing commercial electronics on biodegradable PCB substrates." In: Proceedings of IEEE 39th International Spring Seminar on Electronics Technology. pp. 37-42. 2016. https://doi.org/10.1109/ISSE.2016.7563157

[68] Varga, N., Bokor, L., Fischer, H.-J. "LDM-based dynamic network discovery and selection for IPv6 mobility management optimization in C-ITS environments." In: Proc. of the 4th International Conference on Models and Technologies for Intelligent Transportation Systems (MTITS), Budapest, Hungary, 2015. pp. 483-490. https://doi.org/10.1109/MTITS.2015.7223298

[69] Varga, N., Bokor, L. "Performance evaluation of mobile medical multimedia transmission over ITS GeoNetworking protocol." In: Proc. of 18th IEEE International Conference on e-Health Networking, Applications and Services (Healthcom), Munich, Germany, Sep. 14-17, 2016. pp. 1-5. https://doi.org/10.1109/HealthCom.2016.7749436

[70] Varga, N., Bokor, L., Piri, E. “A network-assisted flow mobility architecture for optimized mobile medical multimedia transmission." Annals of Telecommunications. 71, pp. 489-502. 2016. https://doi.org/10.1007/s12243-016-0501-3

[71] Faigl, Z., Bokor, L., Neves, P. M., Pereira, R. A., Daoud, K., Herbelin, Ph. "Evaluation and Comparison of Signaling Protocol Alternatives for the Ultra Flat Architecture." In: Proc. of the Fifth International Conference on Systems and Networks Communications (ICSNC'10), Nice, France, Aug. 22-27, 2010. pp. 326-334. https://doi.org/10.1109/ICSNC.2010.58

[72] Koscher, K., Czeskis, A., Roesner, F., Patel, S., Kohno, T., Checkoway, S., McCoy, D., Kantor, B., Anderson, D., Shacham, H., Savage, S. "Experimental Security Analysis of a Modern Automobile.” In: IEEE Symposium on Security and Privacy, May, 16-19, 2010. pp. 447-462. https://doi.org/10.1109/SP.2010.34

[73] Checkoway, S., McCoy, D., Kantor, B., Anderson, D., Shacham, H., Savage, S. "Comprehensive Experimental Analyses of Automotive Attack Surfaces." In: SEC'11 Proceedings of the 20th USENIX conference on Security, San Francisco, CA, Aug. 08-12, 2011. 6 p.

[74] Greenberg, A. "Hackers Remotely Kill a Jeep on the Highway - With Me in It." Wired Magazin. July 21, 2015.

[75] Miller, C., Valasek, C. "Adventures in Automotive Networks and Control Units.” Technical White Paper, IOActive, 2014.

[76] Szijj, A., Buttyán, L., Szalay, Zs. "Hacking cars in the style of Stuxnet." Hacktivity, 2015. 
[77] Belső, Z., Gáti, B., Koller, I., Rucz, P., Turóczi, A. "Design of a nonlinear state estimator for navigation of autonomous aerial vehicles." Repüléstudományi Közlemények. XXVII (2015/3), pp. 255-276. 2015.

[78] Rucz, P., Belső, Z., Gáti, B., Koller, I., Turóczi, A. "Design and implementation of nonlinear control systems for rotary and fixed wing UAVs." Repüléstudományi Közlemények. XXVII (2015/3), pp. 277-299. 2015.

[79] Wiandt, B., Kokuti, A., Simon, V. "Application of Collective Movement in Real Life Scenarios: Overview of Current Flocking Solutions." Scalable Computing: Practice and Experience. 16 (3), pp. 233-248. 2015.

[80] Kokuti, A., Simon, V., Wiandt, B.: „Token-based Autonomous Task Allocation in Flocking Systems." In: Federated Conference on Computer Science and Information Systems, Gdansk, Poland, Sep. 11-14, 2016. pp 1501-1506.

[81] Bilicz, S. "Sparse grid surrogate models for electromagnetic problems with many parameters." IEEE Transactions on Magnetics. 52(3), Article No. 6200204, 4 p. 2016. https://doi.org/10.1109/TMAG.2015.2477241

[82] Cai, C., Bilicz, S., Rodet, T., Lambert, M., Lesselier, D. "Metamodelbased nested sampling for model selection in eddy-current testing." IEEE Transactionson Magnetics. 53(4), Article No. 6200912, 12 p. 2017. https://doi.org/10.1109/TMAG.2016.2635626

[83] Vértesy, G., Gasparics, A., Pávó, J., Gyimóthy, S. "Detection of low density magnetic nanoparticles by Fluxset type magnetic probe." International Journal of Applied Electromagnetics and Mechanics. 52 (1-2), pp. 453-460. 2016. https://doi.org/10.3233/JAE-162209

[84] Bilicz, S., Gyimóthy, S., Pávó, J., Tóth, L. L., Badics, Z., Bálint, B. "Modeling of resonant wireless power transfer with integral formulations in heterogeneous media." IEEE Transactions on Magnetics. 52 (3), 7205904, 4 p. 2016. https://doi.org/10.1109/TMAG.2015.2496728

[85] Bilicz, S., Badics, Z., Gyimóthy, S., Bálint, B., Pávó, J. "Modeling of dense windings for resonant wireless power transfer by an integral equation formulation." In: The 17th Biennial IEEE Conference on Electromagnetic Field Computation (CEFC 2016), 2016.

[86] Badics, Z., Bilicz, S., Gyimóthy, S., Pávó, J. "Finite element-integral equation full-wave multi-solver for efficient modeling of resonant wireless power transfer." IEEE Transactions on Magnetics. 52 (3), Article No. 7207904, 4 p. 2016. https://doi.org/10.1109/TMAG.2015.2479402

[87] Bilicz, S., Pávó, J., Gyimóthy, S., Badics, Z. "An integral equation formulation with global series expansion for resonant wireless power transfer." In: 17th International IGTE Symposium on Numerical Field Calculation in Electrical Engineering, Graz, Austria, 2016.

[88] Bilicz, S., Gyimóthy, S., Pávó, J., Horváth, P., Marák, K. "Uncertainty quantification of wireless power transfer systems." In: 2016 IEEE Wireless Power Transfer Conference (WPTC), Aveiro, Portugal, May 5-6, 2016. https://doi.org/10.1109/WPT.2016.7498861

[89] Gyimóthy, S., Bilicz, S., Bálint, B., Pávó, J., Horváth, P. "Visualization of steady-state power flow in resonant WPT systems." In: 17th International IGTE Symposium on Numerical Field Calculation in Electrical Engineering, Graz, Austria, 2016.

[90] Horváth, B., Badics, Z., Pávó, J., Horváth, P. "Validation of numerical models of portable wireless devices for near-field simulation." In: The 17th Biennial IEEE Conference on Electromagnetic Field Computation (CEFC 2016), Miami, FL, USA, Nov. 13-16, 2016. https://doi.org/10.1109/CEFC.2016.7816012

[91] Pető, T., Bilicz, S., Szűcs, L., Gyimóthy, S., Pávó, J. “The radar cross section of small propellers on unmanned aerial vehicles." In: 2016 10th European Conference on Antennas and Propagation (EuCAP), Davos, Switzerland, Apr. 10-15, 2016.

https://doi.org/10.1109/EuCAP.2016.7481645
[92] Szalay, Z. A., Nagy, L. "Indoor Positioning Using Linearly and Circularly Polarized Antennas." Periodica Polytechnica-Electrical Engineering and Computer Science. 60(1), pp. 60-64, 2016.

https://doi.org/10.3311/PPee.9042

[93] Szabó, Z. "Closed Form Kramers-Kronig Relations to Extract the Refractive Index of Metamaterials." IEEE Transactions on Microwave Theory and Techniques. 65 (4), pp. 1150-1159. 2017. https://doi.org/10.1109/TMTT.2016.2633345

[94] Kalvach, A., Szabó, Z. "Calculation of the Image of Extended Objects Placed behind Metamaterial Slabs." Progress In Electromagnetics Research M. 47, pp. 111-120. 2016. https://doi.org/10.2528/PIERM15121006

[95] Kalvach, A., Szabó, Z. "Aberration-free flat lens design for a wide range of incident angles." Journal of the Optical Society of America B. 33(2), pp. A66-A71. 2016. https://doi.org/10.1364/JOSAB.33.000A66

[96] Szabó, Z., Füzi, J. "Implementation and identification of Preisach type hysteresis models with Everett Function in closed form." Journal of Magnetism and Magnetic Materials. 406, pp. 251-258. 2016. https://doi.org/10.1016/j.jmmm.2016.01.027

[97] Pető, T., Rudolf, S. “Quad Channel DVB-T Based Passive Radar.” In: International Radar Symposium (IRS), Krakow, Poland, May 10-12, 2016. https://doi.org/10.1109/IRS.2016.7497383

[98] Szüllö, Á., Seller, R. "Maneuvering target tracking in wide area multilateration radar system." In: International Radar Symposium (IRS), Krakow, Poland, May 10-12, 2016. https://doi.org/10.1109/IRS.2016.7497345

[99] Dudás, L., Gschwindt, A. "The Communication and Spectrum Monitoring System of Smog-1 PocketQube Class Satellite." In: International Conference on Microwave, Radar and Wireless Communications (MIKON), Krakow, Poland, May 9-11, 2016. https://doi.org/10.1109/MIKON.2016.7491999

[100] Fehér, G., Udvary, E. "VLC-Based Indoor Localization." In: Optical Wireless Communications: An Emerging Technology. pp. 609-622. Springer International Publishing, Switzerland (Signals and Communication Technology). 2016.

https://doi.org/10.1007/978-3-319-30201-0_28

[101] Al-Musawi, H. K., Cseh, T., Bohata, J., Pesek, P., Ng, W. P., Ghassemlooy, Z., Udvary, E., Berceli, T., Zvanovec, S. "Experimental optimization of the hybrid RoMMF-FSO system using mode filtering techniques." In: IEEE International Conference on Communications Workshops, ICC, Kuala Lumpur, Malaysia, May, 23-27, 2016. https://doi.org/10.1109/ICCW.2016.7503821

[102] Udvary, E. "Off-set Filtering Effect in SOA Based Optical Access Network." Radioengineering. 25(1), pp. 26-33. 2016. https://doi.org/10.13164/re.2016.0026

[103] Fekete, G., Berceli, T. "Distortions and Their Effect on Signal Transmission in Coherent-OFDM Systems." In: Springer Proceedings in Physics, Vol. 177. pp. 3-17. Springer International Publishing, Switzerland. 2016. https://doi.org/10.1007/978-3-319-27321-1_1

[104] Parajuli, H. N., Udvary, E. "Optimization of optical carrier to sideband ratio for the concatenated AM-PM based optical single sideband radio over fiber system." In: 21 st European Conference on Networks and Optical Communications (NOC), Lisbon, Portugal, Jun. 1-3, 2016. https://doi.org/10.1109/NOC.2016.7507014

[105] Nagy, A., Cseh, T., Szalay, Z., Berceli, T. "Integrated microstrip diplexers for Radio over Fiber." In: 21st International Conference on Microwave, Radar and Wireless Communications (MIKON), Krakow, Poland, May 9-11, 2016. https://doi.org/10.1109/MIKON.2016.7492041

[106] Fekete, G., Mészáros, G., Berceli, T., Udvary, E. "Stability Problems of High Frequency Signals Generated by Direct Mixing Two Laser Beams." Periodica Polytechnica Electrical Engineering and Computer Science. 60(1), pp. 82-87. 2016. https://doi.org/10.3311/PPee.9043 
[107] Schranz, Á., Udvary, E., Kis, Z. "Photon statistics determination for single photon based quantum key distribution." In: 18th International Conference on Transparent Optical Networks (ICTON), Trento, Italy, July 10-14, 2016. https://doi.org/10.1109/ICTON.2016.7550483

[108] Csurgai-Horváth, L., Rieger, I., Kertész, J. "A Survey of the DVB-T Spectrum: Opportunities for Cognitive Mobile Users." Mobile Information Systems. Article ID 3234618, 11 p. 2016. https://doi.org/10.1155/2016/3234618

[109] Csurgai-Horváth, L., Adjei-Frimpong, B. "Annual Statistics of the Alphasat Ka and Q-band Propagation Channel in Budapest, Hungary.” In: The 11th European Conference on Antennas and Propagation (EuCAP 2017), Paris, France, 2017.

[110] Kiss, B., Szirmay-Kalos, L. "Proceedings of the Workshop on the Advances of Information technology." 142 p. BME IIT, Budapest. 2017.

[111] Várady, T., Salvi, P., Karikó, G. “A multi-sided Bézier patch with a simple control structure." Computer Graphics Forum. 35(2), pp. 307-317. 2016. https://doi.org/10.1111/cgf.12833

[112] Kovács, I., Várady, T., Salvi, P. “Applying geometric constraints for perfecting CAD models in reverse engineering." Graphical Models. 82, pp. 44-57. 2015. https://doi.org/10.1016/j.gmod.2015.06.002

[113] Szirmay-Kalos, L., Kacsó, A. "Regularizing Direct Parametric Reconstruction for Dynamic PET with the Method of Sieves.” In: Molecular Imaging Congress. p. M16D-1. 2016.

[114] Szirmay-Kalos, L., Georgiev, I., Magdics, M., Molnár, B., Légrády, D. "Unbiased Light Transport Estimators for Inhomogeneous Participating Media." Computer Graphics Forum. 36 (2), 2017.

[115] Csébfalvi, B., Rácz, G. "Retailoring Box Splines to Lattices for Highly Isotropic Volume Representations.” Computer Graphics Forum. 35(3), pp. 411-420. 2016. https://doi.org/10.1111/cgf.12917

[116] Tóth, M., Ruskó, L., Csébfalvi, B. “Automatic recognition of anatomical regions in three-dimensional medical images." Computers in Biology and Medicine. 76, pp. 120-133. 2016.

https://doi.org/10.1016/j.compbiomed.2016.06.018

[117] Taliga, M., Balla, K. "Risk Management in Medical Software." In: $7^{\text {th }}$ International Multiconference on Complexity, Informatics and Cybernetics. Orlando: IEEE. 2016.

[118] Taliga, M., Balla, K. "BDD Integration Tests to Reach Quality Goals". In: Proceedings of the Workshop on the Advances of Information Technology, Budapest. pp. 11-14. 2017.

[119] Benyo, B., Chase, J., Moeller, K. Desaive, T. “eTime: Engineering Technology-based Innovation in Medicine”. EU FP7, 2013. Retrieved January $31,2017$.

[120] Stewart, K. W., Pretty, C. G., Tomlinson, H., Thomas, F. L., Homlok, J., Szabó Némedi, N., Illyés, A., Shaw, G. M., Benyó, B., Chase, J. G. "Safety, efficacy and clinical generalization of the STAR protocol: a retrospective analysis.” Annals of Intensive Care. 6(1), Article ID 24. 2016. https://doi.org/10.1186/s13613-016-0125-9

[121] Stewart, K., Pretty, C. G., Thomas, F., Shaw, G. M., Desaive, T., Benyo, B., Homlok, J., Illyés, A., Szabó Némedi, N., Chase, J. G. "Generalizability of a Nonlinear Model-based Glycemic Controller." IFAC-PapersOnLine. 49(5), pp. 212-217. 2016. https://doi.org/10.1016/j.ifacol.2016.07.115

[122] Homlok, J., Szlávecz, Á., Stewart, K., Illyés, A., Chase, G. J., Benyó, B. "The effects of ICU specific nutrition management, as a human factor by using Stochastic Targeted glycaemic control." IFAC-PapersOnLine. 49(5), pp. 236-242. 2016. https://doi.org/10.1016/j.ifacol.2016.07.119

[123] Paláncz, B., Stewart, K., Homlok, J., Pretty, C. G., Chase, J. G., Benyó, B. "Stochastic Simulation and Parameter Estimation of the ICING Model.” IFAC-PapersOnLine. 49(5), pp. 218-223. 2016. https://doi.org/10.1016/j.ifacol.2016.07.116
[124] Kannangara, D. O., Newberry, F., Howe, S., Major, V., Redmond, D., Szlavecz, A., Chiew, Y. S., Pretty, C., Benyo, B., Shaw, G. M., Chase, J. G. "Estimating the true respiratory mechanics during asynchronous pressure controlled ventilation." Biomedical Signal Processing and Control. 30, pp. 70-78. 2016. https://doi.org/10.1016/j.bspc.2016.06.014

[125] Szlavecz, A., Chiew, Y., Redmond, D., Beatson, A., Glassenbury, D., Corbett, S., Major, V., Pretty, C., Shaw, G. M., Benyó, B., Desaive, T., Chase, J. "The Clinical Utilisation of Respiratory Elastance Software (CURE Soft): a bedside software for real-time respiratory mechanics monitoring and mechanical ventilation management." BioMedical Engineering OnLine. 13 (1), pp. 140. 2014.

https://doi.org/10.1186/1475-925X-13-140

[126] Szemenyei, M., Vajda, F. "Dimension Reduction for Structured Composite Classes in Multi-Object Environments." In: 15th International Conference on Artificial Intelligence, Knowledge Engineering and Data Bases, Venice. (WSEAS (Ed.)), pp. 134-141. 2016.

[127] Szemenyei, M., Vajda, F. "Dimension Reduction for Objects Composed of Vector Sets." International Journal of Applied Mathematics and Computer Science. 27 (1), pp. 169-180. 2017. https://doi.org/10.1515/amcs-2017-0012

[128] Arató, P., Drexler, D., Rácz, G. “Analyzing the Effect of Decomposition Algorithms on the Heterogeneous Multiprocessing Architectures in System Level Synthesis." Scientific Bulletin of Politechnica University of Timisoara Transactions on Automatic Control and Computer Science. 60(74), pp. 39-46. 2015.

[129] Suba, G., Arató, P. “A new data flow graph model extended for handling loops and mutable data in high level synthesis." In: Workshop on the Advances of Information Technology, BME IIT. 2017.

[130] Lantos, B., Max, G. "Hierarchical Control of Unmanned Ground Vehicle Formations using Multi-Body Approach.” Acta Polytechnica Hungarica. 13(1), pp. 137-156. 2016. https://doi.org/10.12700/APH.13.1.2016.1.10

[131] Peter, G., Kiss, B., Kovacs, G. "Kalman filter based cooperative landmark localization in indoor environment for mobile robots.” In: 2016 IEEE International Conference on Systems, Man, and Cybernetics (SMC), Budapest, Hungary, Oct. 9-12, 2016. pp. 001888-001893. https://doi.org/10.1109/SMC.2016.7844514

[132] Kim, J., Kiss, B., Lee, D. “An adaptive unscented Kalman filtering approach using selective scaling.” In: 2016 IEEE International Conference on Systems, Man, and Cybernetics (SMC), Budapest, Hungary, Oct. 9-12, 2016. pp. 000784-000789. https://doi.org/10.1109/SMC.2016.7844337

[133] Jobbágy, Á., Schultheisz J., Horváth, M., Vraskóné, Réfy H. “Development of an Effective Therapy and Objective Assessment for Children with Birth Injuries." International Journal of Rehabilitation research. 39(4), pp. 354-360. 2016. https://doi.org/10.1097/MRR.0000000000000179

[134] Jobbágy, Á., Schultheisz, J., Horváth, M., Bacsó, P., Csuhaj, P., Vraskó, H. R. "Objective Assessment of Children with Birth Injuries.” In: MEDICON 2016, IFMBE Proceedings, Vol. 57. pp. 571-575. 2016 https://doi.org/10.1007/978-3-319-32703-7_111

[135] Csordás, P., Jobbágy, Á. "Shape analysis of oscillometric pulses.” In: $6^{\text {th }}$ European Conf. of IFMBE, IFMBE Proceedings Vol. 45. pp. 427-430. 2015. https://doi.org/10.1007/978-3-319-11128-5_107

[136] Vincze, D., Kovácsházy, T. "Benchmark Tool for the Characterization of the Real-Time Performance of Linux on System on a Chip Platforms for Measurement Systems.” In: 21 ${ }^{\text {st }}$ IMEKO TC-4 Int. Symposium and 19th Int. Workshop on ADC Modelling and Testing, Budapest, Hungary, Sep. 7-9, 2016. pp. 107-112. 2016.

[137] Dülk, I. "Temperature Solution in Composites by Domain Decomposition." Composite Structures. 147, pp. 54-64. 2016. https://doi.org/10.1016/j.compstruct.2016.03.023 
[138] Kovácsházy, T. “Towards a Quantization Based Accuracy and Precision Characterization of Packet-Based Time Synchronization.” In: Precision Clock Synchronization for Measurement, Control, and Communication (ISPCS), Stockholm, Sweden, Sep. 4-9, 2016. pp. 72-77. 2016. https://doi.org/10.1109/ISPCS.2016.7579512

[139] Sujbert, L., Orosz, Gy. "FFT-based Spectrum Analysis in the Case of Data Loss." IEEE Transactions on Instrumentation and Measurement. 65 (5), pp. 968-976. 2016. https://doi.org/10.1109/TIM.2015.2508278

[140] Sujbert, L., Orosz, Gy. "FFT-based Identification of Data Loss Models." In: 21st IMEKO TC-4 Int. Symposium and 19th Int. Workshop on ADC Modelling and Testing, Budapest, Hungary. pp. 146-151. 2016.

[141] Renczes, B., Kollár, I., Moschitta, A., Carbone, P. "Numerical Optimization Problems of Sine Wave Fitting Algorithms in the Presence of Roundoff Errors." IEEE Transactions on Instrumentation and Measurement. 65 (8), pp. 1785-1795. 2016. https://doi.org/10.1109/TIM.2016.2562218

[142] Renczes, B., Kollár, I., Dabóczi, T. "Efficient Implementation of Least Squares Sine Fitting Algorithms.” IEEE Transactions on Instrumentation and Measurement. 65(12), pp. 2717-2724. 2016. https://doi.org/10.1109/TIM.2016.2600998

[143] Virosztek, T., Kollár, I. "Parameterization of Nonlinearity for Efficient Estimation in ADC Testing." In: 21 st IMEKO TC-4 Int. Symposium and 19th Int. Workshop on ADC Modelling and Testing, Budapest, Hungary, 2016.

[144] Belloch, J. A., Bank, B., Igual, F. D., Quintana-Ortí, E. S., Vidal, A. M. "Solving Weighted Least Squares (WLS) Problems on ARM-based Architectures." The Journal of Supercomputing. 73(1), pp. 530-542. 2017. https://doi.org/10.1007/s11227-016-1910-9

[145] Heikkilä, T., Dobrowiecki, T., Dalgaard, L. "Dealing with Configurability in Robot Systems.” In: MESA 2016, The 12th IEEE/ASME Int. Conf. on Mechatronic and Embedded Systems and Applications, Aucklan, New Zealand, Aug. 29-31, 2016. https://doi.org/10.1109/MESA.2016.7587120

[146] Mészáros, T., Dobrowiecki, T., “Agent-based Reconfigurable Natural Language Interface to Robots.” In: ICAART 2017, Int. Conf. on Agents and Artificial Intelligence, Porto, Portugal, Feb. 24-26, 2017.

[147] Horvath, A., Wolf, P., Nagy, J., Kelemen, A., Horvath, G., Hadhazi, D., Horvath, A., Czetenyi, B., Sutto, Z., Szondy, K. “Overview of a Digital Tomosynthesis Development: New Approaches for Low-dose Chest Imaging”. Radiation Protection Dosimetry. 169(1-4), pp. 171-176. 2016. https://doi.org/10.1093/rpd/ncv469

[148] Horváth, Á. Hadházi, D., Horváth, G. “Analysis of Primary Modulation Based X-ray Scatter Correction Methods.” Medical Physics. 43 (11), pp. 5794-5807. 2016. https://doi.org/10.1118/1.4963209

[149] Marx, P., Millinghoffer, A., Juhász, G., Antal, P. “Joint Bayesian Modelling of Internal Dependencies and Relevant Multimorbidities of a Heterogeneous Disease." Proceedings of Machine Learning Research. 52, pp. 310-320. 2016.

[150] Bolgár, B., Antal, P. "Bayesian Matrix Factorization with Non-Random Missing Data using Informative Gaussian Process Priors and Soft Evidences." Proceedings of Machine Learning Research. 52, pp. 25-36. 2016.

[151] Juhasz, G., Csepany, E., Magyar, M., Edes, A., Eszlari, N., Hullam, G., Antal, P., Kokonyei, Gy., Anderson, I. M., Deakin, J. F. W., Bagdy, Gy. "Variants in the CNR1 Gene Predispose to Headache with Nausea in the Presence of Life Stress." Genes, Brain and Behavior. 16 (3), pp. 384-393. 2017. https://doi.org/10.1111/gbb.12352

[152] Hegyi, M., Arany, A., Semsei, A. F., Csordas, K., Eipel, O., Gezsi, A., Kutszegi, N., Csoka, M., Muller, J., Erdelyi, D. J., Antal, P., Szalai, C., Kovacs, G. T. "Pharmacogenetic Analysis of High-Dose Methotrexate Treatment in Children with Osteosarcoma." Oncotarget. 8 (6), pp. 93889398. 2017. https://doi.org/10.18632/oncotarget.11543
[153] Bergmann, G., Debreceni, C., Ráth, I., Varró, D., “Query-based Access Control for Secure Collaborative Modeling using Bidirectional Transformations." In: ACM/IEEE 19th Int. Conf. on Model Driven Engineering Languages and Systems, (ACM Distinguished Paper Award). pp. 351361. 2016. https://doi.org/10.1145/2976767.2976793

[154] Darvas, D., Majzik, I., Blanco Viñuela, E., "Formal Verification of Safety PLC Based Control Software.” Integrated Formal Methods. 9681, pp. 508-522. 2016. https://doi.org/10.1007/978-3-319-33693-0_32

[155] Dávid, I., Ráth, I., Varró, D. "Foundations for Streaming Model Transformations by Complex Event Processing." Software \& Systems Modeling. 28 p. 2016. https://doi.org/10.1007/s10270-016-0533-1

[156] Gönczy, L., Majzik, I., Bozóki, Sz., Pataricza, A. "MDD-Based Design, Configuration, and Monitoring of Resilient Cyber-Physical Systems." In: Trustworthy Cyber-Physical Systems Engineering. pp. 395-420. CRC Press, Boca Raton, FL. 2016. https://doi.org/10.1201/9781315369105-16

[157] Hajdu, Á., Tóth, T., Vörös, A., Majzik, I. “A Configurable CEGAR Framework with Interpolation-Based Refinements.” In: Formal Techniques for Distributed Objects, Components, and Systems. Vol. 9688, Springer, pp. 158-174. 2016. https://doi.org/10.1007/978-3-319-39570-8_11

[158] Kocsis, I., Tóth, Á., Szatmári, Z., Dabóczi, T., Pataricza, A., Guta, G. "Towards Cyber-Physical System Technologies over Apache VCL." International Journal of Cloud Computing. 5(1-2), pp. 91-111. 2016. https://doi.org/10.1504/IJCC.2016.075101

[159] Marussy, K., Klenik, A., Molnár, V., Vörös, A., Majzik, I., Telek, M. “Efficient Decomposition Algorithm for Stationary Analysis of Complex Stochastic Petri Net Models." In: Application and Theory of Petri Nets and Concurrency. Vol. 9698, Springer. pp. 281-300. 2016. https://doi.org/10.1007/978-3-319-39086-4_17

[160] Molnár, V., Vörös, A., Darvas, D., Bartha, T., Majzik, I. "Componentwise Incremental LTL Model Checking." Formal Aspects of Computing. 28 (3), pp. 345-379. 2016. https://doi.org/10.1007/s00165-015-0347-x

[161] Semeráth, O., Vörös, A., Varró, D. "Iterative and Incremental Model Generation by Logic Solvers.” In: 19th International Conference on Fundamental Approaches to Software Engineering. Springer-Verlag, New York. pp. 87-103. 2016. https://doi.org/10.1007/978-3-662-49665-7_6

[162] Varró, D., Bergmann, G., Hegedüs, Á., Horváth, Á., Ráth, I., Ujhelyi, Z. "Road to a Reactive and Incremental Model Transformation Platform: Three Generations of the VIATRA Framework." Software \& Systems Modeling. 15 (3), pp. 609-629. 2016. https://doi.org/10.1007/s10270-016-0530-4

[163] Bateman, M., Katz, N. H. "New bounds on cap sets." Journal of the American Mathematical Society. 25, pp. 585-613. 2012. https://doi.org/10.1090/S0894-0347-2011-00725-X

[164] Bernholt, T., Gülich, A., Hofmeister, T., Schmitt, N. "Football elimination is hard to decide under the 3-point-rule." In: Lecture Notes in Computer Science. Vol. 1672, pp. 410-418. 1999. https://doi.org/10.1007/3-540-48340-3_37

[165] Cechlárová, K., Potpinková, E., Schlotter, I. "Refining the complexity of the sports elimination problem." Discrete Applied Mathematics. 199, pp. 172-186. 2016. https://doi.org/10.1016/j.dam.2015.01.021

[166] Schlotter, I., Cechlárová, K. “A connection between sports and matroids: How many teams can we beat?" Algorithmica. First Online, 21 p. 2017. https://doi.org/10.1007/s00453-016-0256-2

[167] Chan, T. H. "On sets of integers, none of which divides the product of $\mathrm{k}$ others.” European Journal of Combinatorics. 32 (3), pp. 443-447. 2011. https://doi.org/10.1016/j.ejc.2010.11.010

[168] Chan, T. H., Györi, E., Sárközy, A. “On a problem of Erdős on integers, none of which divides the product of $k$ others." European Journal of Combinatorics. 31(1), pp. 260-269. 2010. https://doi.org/10.1016/j.ejc.2009.02.005 
[169] Croot, E., Lev, V. F., Pach, P. P. "Progression-free sets in $\mathrm{Z}_{4}^{\mathrm{n}}$ are exponentially small." Annals of Mathematics. 185(1), pp. 331-337. 2017. https://doi.org/10.4007/annals.2017.185.1.7

[170] Downey, R. G., Fellows, M. R. "Fundamentals of Parameterized Complexity. Texts in Computer Science." 763 p. Springer-Verlag, London. 2013. https://doi.org/10.1007/978-1-4471-5559-1

[171] Ellenberg, J. S., Gijswijt, D. "On large subsets of $F_{q}{ }^{n}$ with no three-term arithmetic progression." Annals of Mathematics. 185(1), pp. 339-343. 2017. https://doi.org/10.4007/annals.2017.185.1.8

[172] Erdős, P. "On sequences of integers no one of which divides the product of two others and on some related problems." Tomsk. Gos. Univ. Uchen. Zap. 2, pp. 74-82. 1938.

[173] Kern, W., Paulusma, D. "The computational complexity of the elimination problem in generalized sports competitions." Discrete Optimization. 1(2), pp. 205-214. 2004. https://doi.org/10.1016/j.disopt.2003.12.003

[174] Meshulam, R. "On subsets of finite abelian groups with no 3-term arithmetic progressions." Journal of Combinatorial Theory, Series A. 71 (1), pp. 168-172. 1995. https://doi.org/10.1016/0097-3165(95)90024-1

[175] Pach, P. P. "Generalized multiplicative Sidon sets." Journal of Number Theory. 157, pp. 507-529. 2015. https://doi.org/10.1016/j.jnt.2015.05.018

[176] Pach, P. P. "Ramsey type results on the solvability of certain equations in Z_m." Integers. 13, 9 p. 2013.

[177] Pach, P. P. "The Ramsey-type version of a problem of Pomerance and Schinzel." Acta Arithmetica. 156(1), pp. 1-5. 2012.

[178] Pach, P. P., Sándor, Cs. "Multiplicative bases and an Erdős problem." Combinatorica. to appear, 2016.

[179] Papp, D., Lovas, D., Szücs, G. "Object Detection, Classification, Tracking and individual Recognition for Sea Images and Videos.” In: Working Notes of CLEF 2016 - Conference and Labs of the Evaluation Forum, Évora, Portugal, Sep. 5-8, 2016. pp. 525-533.

[180] Barta, G., Nagy, G., Papp, G., Simon, G. "Forecasting framework for open access time series in energy." In: IEEE International Energy Conference (ENERGYCON) 2016, Leuven, Belgium, Apr. 4-8, 2016. 6 p. https://doi.org/10.1109/ENERGYCON.2016.7514015

[181] Nagy, G., Barta, G., Kazi, S., Borbély, Gy., Simon, G. “Probabilistic solar and wind power forecasting using a generalized additive tree ensemble approach." INTERNATIONAL JOURNAL OF FORECASTING. 32(3), pp. 1087-1093. 2016. https://doi.org/10.1016/j.ijforecast.2015.11.013

[182] Biczok, G., Dramitinos, M., Toka, L., Heegaard, P. E., Lonsethagen, H. "Manufactured by software: SDN-enabled multi-operator composite services with the 5G Exchange." IEEE Communications Magazine. 55(4), pp. 80-86. 2017. https://doi.org/10.1109/MCOM.2017.1600197

[183] Öllös, G., Vida, R. "Adaptive Multiresolution Sampling in Event-driven WSNs.” Telecommunication Systems. 61(2), pp. 337-347. 2016. https://doi.org/10.1007/s11235-015-0005-x

[184] Toka, L., Lajtha, B., Hosszú, E., Beleczki, A., Godor, I. "The difference between leisure and competitive squash.” In: KDD workshop on LargeScale Sports Analytics, San Francisco, Aug. 14-18, 2016.

[185] Guerzoni, R., Vaishnavi, I., Caparros, D. P., Galis, A., Tusa, F., Monti, P., Sganbelluri, A., Biczok, G., Sonkoly, B., Toka, L., Ramos, A., Melián, J., Dugeon, O., Cugini, F., Martini, B., Iovanna, P., Giuliani, G., Figueiredo, R., Contreras-Murillo, L. M., Bernardos, C. J., Santana, C., Szabo, R. "Analysis of end-to-end multi-domain management and orchestration frameworks for software defined infrastructures: an architectural survey." Transactions on Emerging Telecommunications Technologies. 28, 19 p. 2017. https://doi.org/10.1002/ett.3103

[186] Molnár, S., Móczár, Z., Sonkoly, B. "Living with Congestion: Digital Fountain based Communication Protocol." Computer Communications. 80, pp. 82-100. 2016. https://doi.org/10.1016/j.comcom.2016.01.008
[187] Bereczky, N., Duch, A., Németh, K., Roura, S. "Quad-kd trees: A general framework for kd trees and quad trees." Theoretical Computer Science. 616, pp. 126-140. 2016. https://doi.org/10.1016/j.tcs.2015.12.030

[188] Castoldi, P., Cugini, F., Cinkler, T. Marino, P. P. "Special issue based on selected IEEE ONDM 2015 papers." Photonic Network Communications. 31(3), pp. 373-375. 2016. https://doi.org/10.1007/s11107-016-0639-y

[189] Simon, Cs., Maliosz, M., Bíró, J., Gerő, B., Kern, A. “5G exchange for iner-domain resource sharing." In: IEEE Symposium on Local and Metropolitan Area Networks (LANMAN), Rome, Italy, June 13-15, 2016. https://doi.org/10.1109/LANMAN.2016.7548842

[190] Rottenstreich, O., Tapolcai, J. "Optimal Rule Caching and Lossy Compression for Longest Prefix Matching." IEEE-ACM Transactions on Networking. 25 (2), pp. 864-878. 2017. https://doi.org/10.1109/TNET.2016.2611482

[191] Pašić, A., Babarczi, P., Körösi, A. "Diversity coding-based survivable routing with QoS and differential delay bounds." Optical Switching and Networking. 23(Part 2), pp. 118-128. 2017. https://doi.org/10.1016/j.osn.2016.06.005

[192] Molnár, L., Pongrácz, G., Enyedi, G., Kis, Z. L., Csikor, L., Juhász, F., Kőrösi, A., Rétvári, G. "Dataplane Specialization for High-performance OpenFlow Software Switching." In: Proceedings of the 2016 ACM SIGCOMM Conference, Florianopolis, Brazil, Aug. 22-26, 2016. pp. 532-552. https://doi.org/10.1145/2934872.2934887

[193] Varga, P., Blomstedt, F., Ferreira, L. L., Eliasson, J., Johansson, M., Delsing, J., Martínez de Soria, I. "Making system of systems interoperable - The core components of the arrowhead framework." Journal of Network and Computer Applications. 81, pp. 85-95. 2017. https://doi.org/10.1016/j.jnca.2016.08.028

[194] Plosz, S., Hegedűs, Cs., Varga, P. “Advanced Security Considerations in the Arrowhead Framework." In: Lecture Notes in Computer Science. Vol. 9923, pp. 234-245. 2016. https://doi.org/10.1007/978-3-319-45480-1_19

[195] Hegedüs, Cs., Kozma, D., Soos, G., Varga, P. "Enhancements of the Arrowhead Framework to Refine Inter-cloud Service Interactions." In: Proceedings of the 42nd Annual Conference of the IEEE Industrial Electronics Society (IECON2016), Florence, Italy, 2016. https://doi.org/10.1109/IECON.2016.7793757

[196] Jantunen, E., Zurutuza, U., Ferreira, L. F., Varga, P. "Optimising maintenance: What are the expectations for Cyber Physical Systems." In: Proceedings of the 3rd International Workshop on Emerging Ideas and Trends in Engineering of Cyber-Physical Systems (EITEC 2016), Vienna, Austria, 2016. pp. 53-58. https://doi.org/10.1109/EITEC.2016.7503697

[197] Orosz, P., Tothfalusi, T., Varga, P. "C-GEP: Adaptive network management with reconfigurable hardware.” In: IEEE/IFIP Int'l Symposium on Integrated Network Management (IM2015), Ottawa, Canada 2015. pp. 954-959. https://doi.org/10.1109/INM.2015.7140417

[198] Varga, P., Kovacs, L., Tothfalusi, T., Orosz, P. “C-GEP: 100 Gbit/s capable, FPGA-based, reconfigurable networking equipment.” In: IEEE 18th International Conference on High Performance Switching and Routing (HPSR2015), Budapest, Hungary, 2015. pp. 76-81. https://doi.org/10.1109/HPSR.2015.7483084

[199] Csapó, T. G., Németh, G., Cernak, M., Garner, P. N. "Modeling Unvoiced Sounds In Statistical Parametric Speech Synthesis with a Continuous Vocoder.” In: Proc. EUSIPCO, Budapest, Hungary, 2016. pp. 1338-1342. https://doi.org/10.1109/EUSIPCO.2016.7760466

[200] Tóth, B. P., Csapó, T. G. "Continuous Fundamental Frequency Prediction with Deep Neural Networks.” In: Proc. EUSIPCO, Budapest, Hungary, 2016, pp. 1348-1352. https://doi.org/10.1109/EUSIPCO.2016.7760468

[201] Nagy, P., Németh, G. „Improving HMM Speech Synthesis of Interrogative Sentences by Pitch Track Transformations." Speech Communication. 82, pp. 97-112. 2016. https://doi.org/10.1016/j.specom.2016.06.005 
[202] Tóth, B. P., Osváth, M., Papp, D., Szűcs, G. “Deep Learning and SVM Classification for Plant Recognition in Content-Based Large Scale Image Retrieval." In: Working Notes of Conference and Labs of the Evaluation Forum, Évora, Portugal, Sep. 5-8, 2016, pp. 569-578.

[203] Tóth, B. P. "Neurális hálózatok: Beszélő számítógépek mély gondolatokkal.”ÉLET ÉS TUDOMÁNY. LXXI, pp. 944-946. 2016. (In Hungarian)

[204] Tulics, M. G., Kazinczi, F., Vicsi, K. "Statistical analysis of acoustical parameters in the voice of children with juvenile dysphonia." In: 18th International Conference on Speech and Computer SPECOM 2016. Budapest, pp. 667-674. 2016. https://doi.org/10.1007/978-3-319-43958-7_81

[205] Sztahó, D, Vicsi, K. "Estimating the severity of Parkinson's disease using voiced ratio and nonlinear parameters.” In: Statistical Language and Speech Processing: 4th International Conference, SLSP 2016, Pilsen, Csehország, Oct. 11-12, 2016. pp. 96-107. https://doi.org/10.1007/978-3-319-45925-7_8

[206] Kiss, G., Tulics, M. G., Sztahó, D., Esposito, A., Vicsi, K. "Language independent detection possibilities of depression by speech." In: Recent Advances in Nonlinear Speech Processing. pp. 103-114. Springer International Publishing, Cham. 2016. https://doi.org/10.1007/978-3-319-28109-4_11

[207] Szaszák, Gy., Beke, A., Olaszy, G., Tóth, B. P. “Gépi beszéd természetességének növelése automatikus, beszédjel alapú hangsúlycímkéző algoritmussal.” In: XII. Magyar Számítógépes Nyelvészeti Konferencia (MSZNY 2016), Szeged, Magyarország, 2016. pp. 144-153. (In Hungarian)

[208] Baranyi, P. "TP model transformation based control design framework." 230 p. Springer International Publishing, 2016. https://doi.org/10.1007/978-3-319-19605-3

[209] Halász, B. G., Németh, B., Cselkó, R., Göcsei, G. “Adaptation of the application of portable protective air gap to the Hungarian live-line maintenance technology.” In: IEEE Electrical Insulation Conference (EIC), Montreal, QC, Canada, 2016. pp. 97-100. IEEE, New York. 2016. https://doi.org/10.1109/EIC.2016.7548576

[210] Halász, B. G., Göcsei, G., Németh, B. "Investigation of terrain effects on PPAG breakdown characteristics.” In: IEEE 13th International Conference on Transmission \& Distribution Construction, Operation \& LiveLine Maintenance. Paper 16ESMO0051. 2016.

[211] Halász, B. G., Németh, B., Göcsei, G. "A special risk during live - line maintenance.” In: Proc. of DIAGNOSTIKA'16 - Conference on Diagnostics in Electrical Engineering CDEE 2016, Pilsen, Czech Republic. Paper ID70. 2016. https://doi.org/10.1109/DIAGNOSTIKA.2016.7736484

[212] Buzási, A., Szalmáné Csete, M., Németh, B. "Smart közlekedés a fenntartható városfejlesztésben." In: Innováció és fenntartható felszíni közlekedés, IFFK 2016. Magyar Mérnökakadémia (MMA), Budapest. 2016. pp. 86-91. (In Hungarian)

[213] Göcsei, G., Németh, B., Cselkó, R., Bokor, T. "Failure analysis and inspection of long term behaviour of selected medium voltage equipment." In: IEEE Electrical Insulation Conference (EIC), Montreal, QC, Canada, 2016. pp. 264-267. IEEE, New York. 2016. https://doi.org/10.1109/EIC.2016.7548709
[214] Göcsei, G., Németh, B. "Current issues regarding to the inspection of conductive clothing." In: IEEE 13th International Conference on Transmission \& Distribution Construction, Operation \& Live-Line Maintenance. Paper 16ESMO0039. 2016.

[215] Göcsei, G., Németh, B., Meixner, J. “A new type of conductor car for high voltage live-line maintenance.” In: IEEE 13th International Conference on Transmission \& Distribution Construction, Operation \& LiveLine Maintenance. Paper 16ESMO0038. 2016.

[216] Göcsei, G., Németh, B. "Shielding of magnetic fields during high voltage live-line maintenance." In: IEEE 13th International Conference on Transmission \& Distribution Construction, Operation \& Live-Line Maintenance. Paper 16ESMO0040. 2016.

[217] Tóth, Z., Kiss, I., Palotai, R. "Problematic of the simulation and modelling in the aspect of lightning protection.” In: 12th Miklós Iványi International PhD \& DLA Symposium: ARCHITECTURAL, ENGINEERING AND INFORMATION SCIENCES ABSTRACT BOOK. p. 117. Pollack Press, Pécs. 2016.

[218] Abdelfatah, A., Raisz, D. "Optimal Charging/Discharging of PHEVs for Predictability Enhancement of Wind Power Generation." Periodica Polytechnica Electrical Engineering and Computer Science. 60(4), pp. 261-265. 2016. https://doi.org/10.3311/PPee.10019

[219] Divényi, D., Polgári, B., Raisz, D., Sleisz, Á., Sőrés, M. P. “Proposal of a New European Cooptimised Energy and Ancillary Service Market Design - Part I - Concept; Part II - Mathematical Model, Case Study and Prototype." In: 13th International Conference on the European Energy Market, Lisbon, Portugal. 2016.

[220] Raisz, D., Mavromatakis, F. "Preface." Periodica Polytechnica Electrical Engineering and Computer Science. 60(4), pp. 245-246. 2016.

[221] Hartmann, B., Farkas, Cs. "Energy efficient data centre infrastructure Development of a power loss model." Energy and Buildings. 127, pp. 692-699. 2016. https://doi.org/10.1016/j.enbuild.2016.06.037

[222] Hartmann, B. “Energiatárolás - költség helyett érték.” Elektrotechnika. 109 (1-2), pp. 5-6. 2016. (in Hungarian)

[223] Horváth, V., Varjú, Gy. "A nagyvasúti villamos vontatási rendszerek." In: Fejezetek az Elektrotechnikából. Magyar Elektrotechnikai Egyesület, Budapest. 2016. (in Hungarian)

[224] Sleisz, Á., Raisz, D. "Complex supply orders with ramping limitations and shadow pricing on the all European day-ahead electricity market." International Journal of Electrical Power and Energy Systems. 83, pp. 26-32. 2016. https://doi.org/10.1016/j.ijepes.2016.03.061 